\title{
Enhanced production of $\gamma$-amino acid 3-amino-4-hydroxybenzoic acid by recombinant Corynebacterium glutamicum under oxygen limitation
}

Hideo Kawaguchi ${ }^{1,2}$, Tomohisa Hasunuma ${ }^{1,2}$, Yasuo Ohnishi ${ }^{3,4}$, Takashi Sazuka ${ }^{5}$, Akihiko Kondo 1,2,6,7*(1) and Chiaki Ogino 2,6

\begin{abstract}
Background: Bio-based aromatic compounds are of great interest to the industry, as commercial production of aromatic compounds depends exclusively on the unsustainable use of fossil resources or extraction from plant resources. Y-amino acid 3-amino-4-hydroxybenzoic acid (3,4-AHBA) serves as a precursor for thermostable bioplastics.

Results: Under aerobic conditions, a recombinant Corynebacterium glutamicum strain KT01 expressing griH and gril genes derived from Streptomyces griseus produced 3,4-AHBA with large amounts of amino acids as by-products. The specific productivity of 3,4-AHBA increased with decreasing levels of dissolved oxygen (DO) and was eightfold higher under oxygen limitation ( $D O=0 \mathrm{ppm}$ ) than under aerobic conditions ( $\mathrm{DO} \geq 2.6 \mathrm{ppm})$. Metabolic profiles during 3,4-AHBA production were compared at three different DO levels $(0,2.6$, and $5.3 \mathrm{ppm})$ using the DO-stat method. Results of the metabolome analysis revealed metabolic shifts in both the central metabolic pathway and amino acid metabolism at a DO of $<33 \%$ saturated oxygen. Based on this metabolome analysis, metabolic pathways were rationally designed for oxygen limitation. An Idh deletion mutant, with the loss of lactate dehydrogenase, exhibited 3.7-fold higher specific productivity of 3,4-AHBA at DO $=0 \mathrm{ppm}$ as compared to the parent strain KT01 and produced $5.6 \mathrm{~g} / \mathrm{L}$ 3,4-AHBA in a glucose fed-batch culture.
\end{abstract}

Conclusions: Our results revealed changes in the metabolic state in response to DO concentration and provided insights into oxygen supply during fermentation and the rational design of metabolic pathways for improved production of related amino acids and their derivatives.

Keywords: Corynebacterium glutamicum, Dissolved oxygen, Metabolic engineering, Metabolome analysis, Amino acid

\footnotetext{
*Correspondence: akondo@kobe-u.ac.jp

${ }^{1}$ Graduate School of Science, Technology and Innovation, Kobe University, 1-1 Rokkodai, Nada, Kobe 657-8501, Japan

Full list of author information is available at the end of the article
}

(c) The Author(s) 2021. Open Access This article is licensed under a Creative Commons Attribution 4.0 International License, which permits use, sharing, adaptation, distribution and reproduction in any medium or format, as long as you give appropriate credit to the original author(s) and the source, provide a link to the Creative Commons licence, and indicate if changes were made. The images or other third party material in this article are included in the article's Creative Commons licence, unless indicated otherwise in a credit line to the material. If material is not included in the article's Creative Commons licence and your intended use is not permitted by statutory regulation or exceeds the permitted use, you will need to obtain permission directly from the copyright holder. To view a copy of this licence, visit http://creativecommons.org/licenses/by/4.0/. The Creative Commons Public Domain Dedication waiver (http://creativeco mmons.org/publicdomain/zero/1.0/) applies to the data made available in this article, unless otherwise stated in a credit line to the data. 


\section{Graphical Abstract}
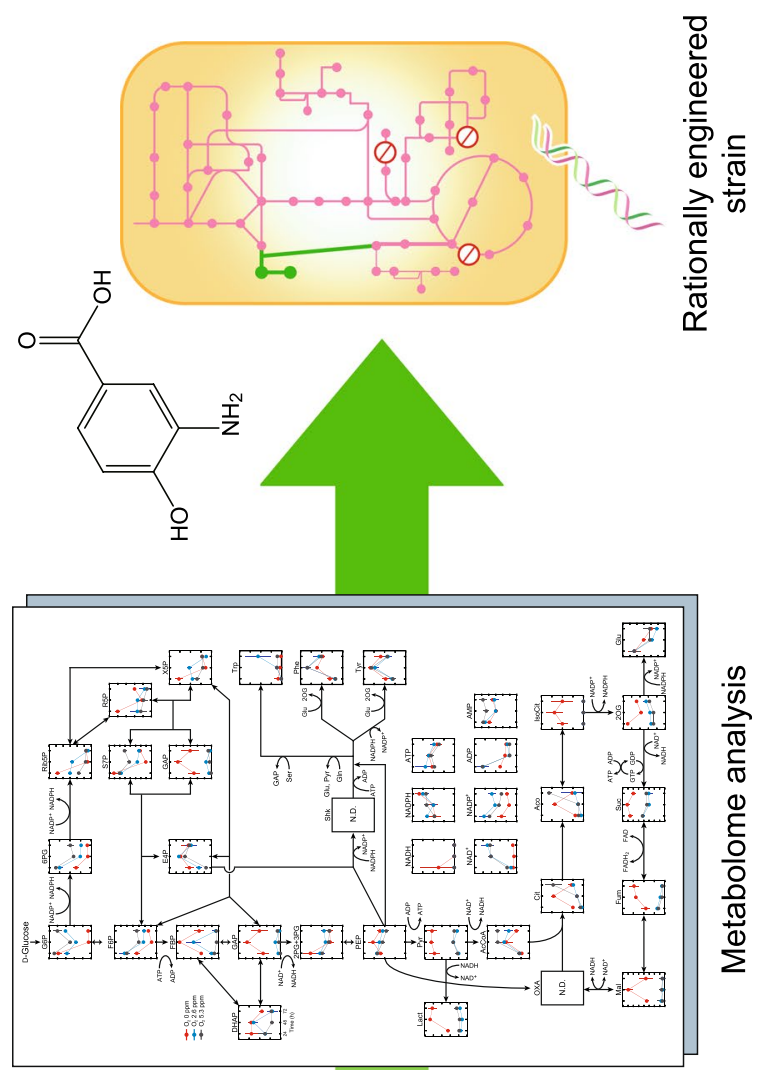

$\frac{00}{0}$
$\frac{2}{0}$
$\frac{1}{0}$
0
$\frac{0}{2}$
0
$\frac{0}{0}$
$\frac{0}{0}$
$\frac{10}{2}$
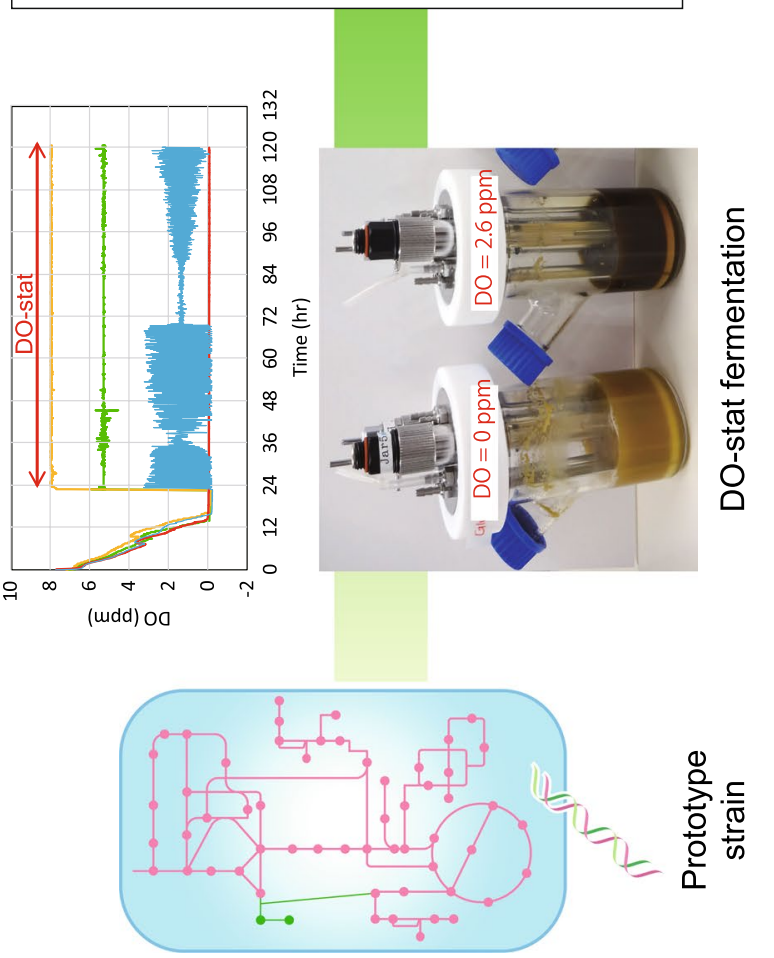


\section{Background}

Bio-based aromatic compounds are of great interest to the industry as commercial production of aromatic compounds depends exclusively on the unsustainable use of fossil resources or extraction from plant resources [1]. Because aromatic compounds are essential precursors for the synthesis of strong, thermostable plastics [2,
3], metabolic engineering of the shikimate pathway for microbial production of aromatic compounds, such as caffeic acid [4], (S)-reticuline [5], salicylate [6], and styrene [7], has been studied since the 2000s. More recently, 3-amino-4-hydroxybenzoic acid (3,4-AHBA), which serves as a precursor for ultra-thermoresistant bioplastics, has been produced from lignocellulosic biomass [8].

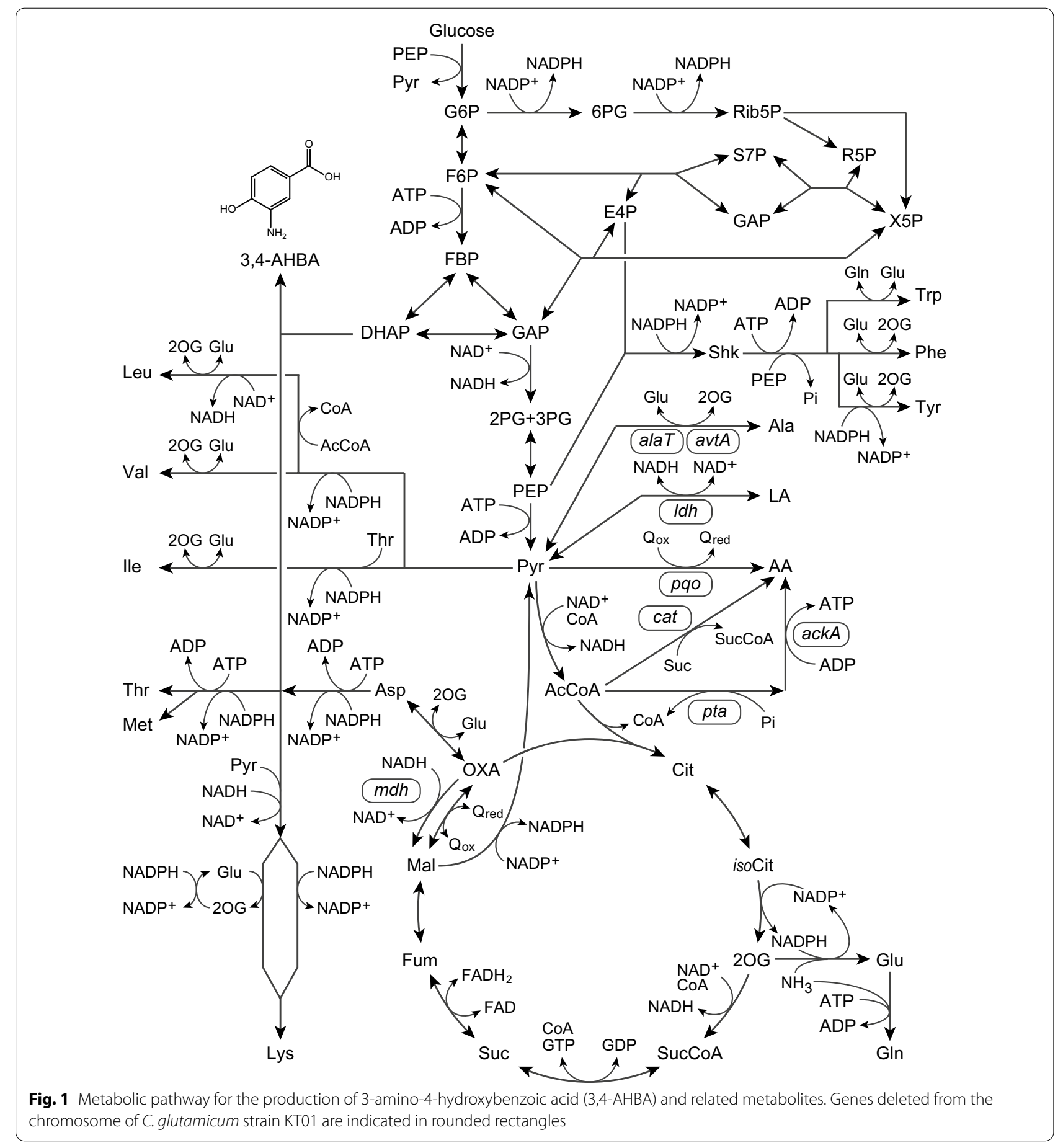


The synthetic pathway of $\gamma$-amino acid 3,4-AHBA differs entirely from the well-known shikimate pathway, which is essential for synthesizing aromatic amino acids, L-phenylalanine (Phe), L-tyrosine (Tyr), and L-tryptophan (Trp) [9]. In a simple two-step reaction (aldol condensation followed by cyclization and aromatization), an aromatic ring of 3,4-AHBA is formed from $\mathrm{C}_{4}$ (L-aspartate-4-semialdehyde) and $\mathrm{C}_{3}$ (dihydroxyacetone phosphate [DHAP]) primary metabolites. In Streptomyces griseus, the enzymes GriI and GriH, encoded by two genes, griI and griH, respectively, catalyze 3,4-AHBA synthesis [10]. A recombinant Corynebacterium glutamicum

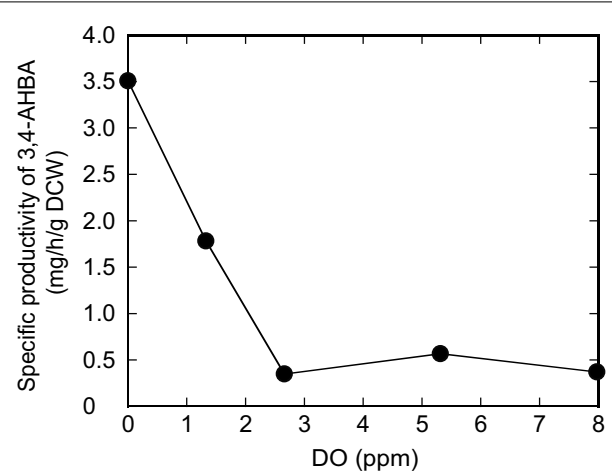

Fig. 2 Effect of dissolved oxygen (DO) concentration on the specific productivity of 3-amino-4-hydroxybenzoic acid (3,4-AHBA). The specific productivity of 3,4-AHBA in recombinant C. glutamicum strain KT01 after $72 \mathrm{~h}$ of cultivation is indicated. The DO-stat method was started after $24 \mathrm{~h}$ of cultivation, and five DO levels $(0,1.3,2.6,5.3$, and $8.0 \mathrm{ppm}$ ) were implemented and controlled by the speed of agitation. The data are presented as averages \pm standard deviation calculated from the results of duplicate independent experiments strain expressing the griH and griI genes successfully produced 3,4-AHBA from sugars [11] (Fig. 1). However, strategies to improve 3,4-AHBA production, including metabolic engineering and bioengineering, need to be identified.

Dissolved oxygen (DO) concentration is one of the most important factors determining the performance of the fermentation process [12]. Unlike anaerobic fermentation of organic acids, the formation of aromatic compounds and amino acids requires oxygen, as reduced nicotinamide adenine dinucleotide phosphate (NADPH) required for the synthesis is primarily supplied through the oxidative pentose phosphate pathway (PPP; Fig. 1) $[13,14]$. For example, the production of aspartate derivatives of L-lysine (Lys) and 1,5-diaminopentane was implemented at a DO level of $\geq 20 \%$ saturated oxygen to regenerate NADPH [15-17]. In addition, aromatic compounds of shikimate and its derivatives have been produced at a DO of $\geq 10 \%$ saturated oxygen level in culture using E. coli and C. glutamicum $[18,19]$.

3,4-AHBA production competes with Lys biosynthesis for the availability of the common precursor L-aspartate-4-semialdehyde derived from aspartate. In glucose metabolism, $2 \mathrm{~mol}$ of NADPH is required to synthesize $1 \mathrm{~mol}$ of 3,4-AHBA, while $4 \mathrm{~mol}$ of NADPH is required to synthesize $1 \mathrm{~mol}$ of Lys [16]. In C. glutamicum, NADPH supply predominantly depends on oxidative PPP, and the increased flux improves Lys yield (Fig. 1) [15, 20]. These suggest that a diminished NAPDH supply under oxygen restriction can direct more carbon from Lys to 3,4AHBA. However, metabolic profiles, including amino acid synthesis under oxygen-limited conditions, have not been extensively studied, although the transcriptome of
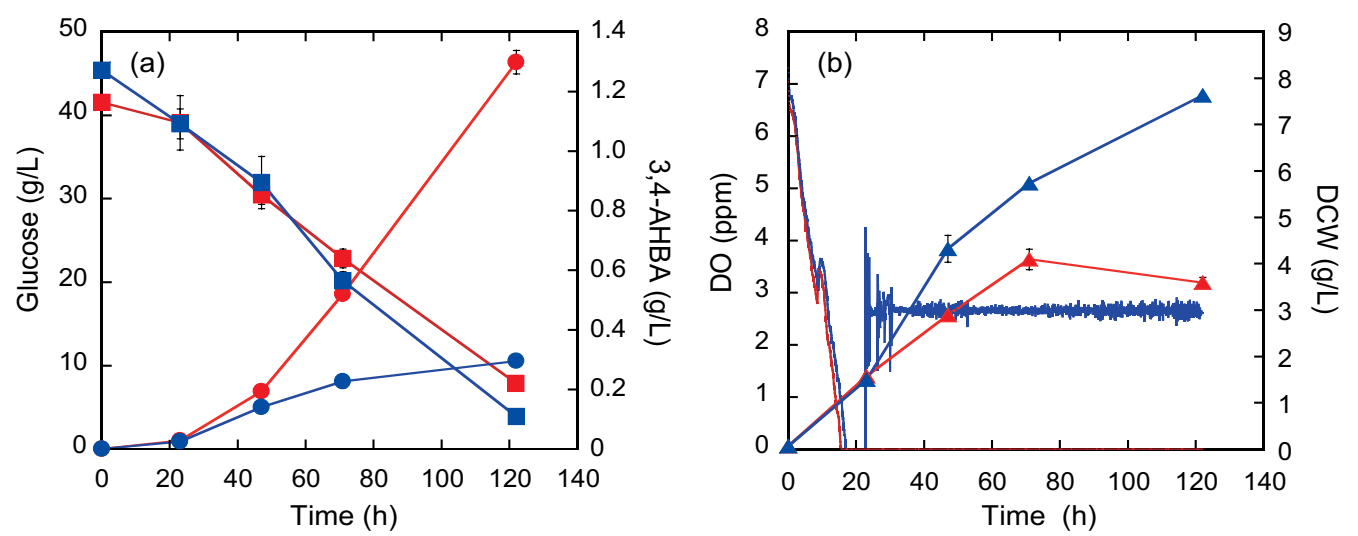

Fig. 3 Dissolved oxygen (DO)-stat cultivation for 3-amino-4-hydroxybenzoic acid (3,4-AHBA) production by recombinant C. glutamicum strain KT01. a The concentrations of glucose (squares), 3,4-AHBA (circles), b dry cell weight (DCW) (triangles), and DO concentration (continuous lines) are indicated. The DO-stat method was started after $24 \mathrm{~h}$ of cultivation, and two DO levels (0 [reds] and 2.6 ppm [blues]) were implemented and controlled by the speed of agitation. The data are presented as averages \pm standard deviation calculated from the results of duplicate independent experiments 
C. glutamicum has been extensively studied under different DO conditions [12, 21-23].

The present study demonstrated enhanced 3,4-AHBA production by recombinant $C$. glutamicum under oxygen limitation in a jar fermentor using the DO-stat program. Using the DO-stat method, metabolic profiles at three different levels of DO were compared, and the metabolome analysis revealed metabolic shifts in both the central metabolic pathway and amino acid metabolism at a threshold DO level. Based on the metabolome analysis results, the metabolic pathway of $C$. glutamicum was rationally designed to tailor to oxygen limitation and thus significantly improve the specific productivity of 3,4-AHBA.

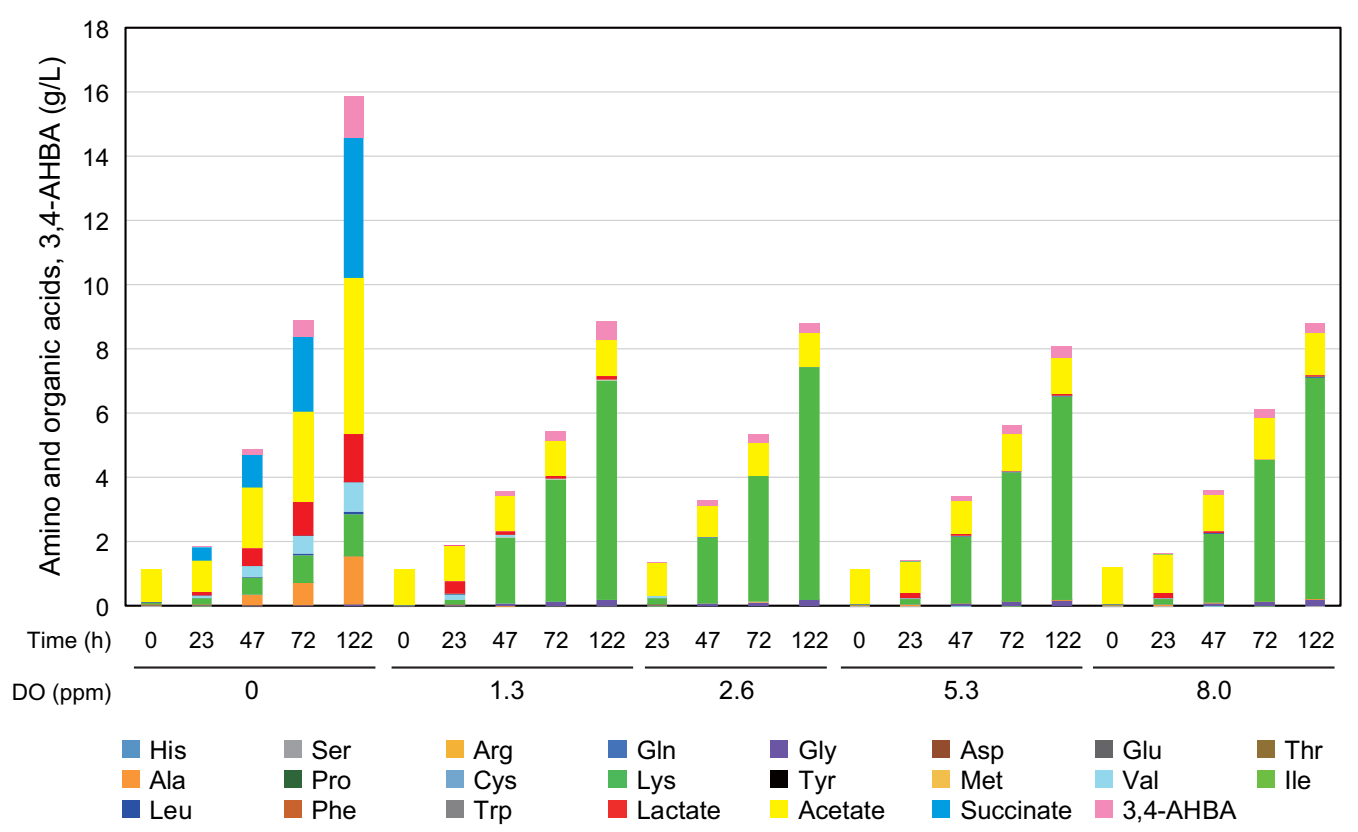

Fig. 4 Effect of dissolved oxygen (DO) concentration on the production of amino acids and organic acids. The DO-stat method was started after $24 \mathrm{~h}$ of cultivation, and five DO levels $(0,1.3,2.6,5.3$, and $8.0 \mathrm{ppm})$ were implemented and controlled by the speed of agitation during 3-amino-4-hydroxybenzoic acid (3,4-AHBA) production by recombinant C. glutamicum strain KT01. The data are presented as averages calculated from the results of duplicate independent experiments

Table 1 Effect of dissolved oxygen (DO) on fermentation products and cell growth

\begin{tabular}{|c|c|c|c|c|c|}
\hline & $\mathrm{DO}$ (ppm) & & & & \\
\hline & 0 & 1.3 & 2.6 & 5.3 & 8.0 \\
\hline 3,4-AHBA $(\mathrm{g} / \mathrm{L})^{\mathrm{a}}$ & $1.30 \pm 0.04$ & $0.61 \pm 0.03$ & $0.30 \pm 0.02$ & $0.37 \pm 0.01$ & $0.30 \pm 0.01$ \\
\hline Total amino acids $(\mathrm{g} / \mathrm{L})^{\mathrm{a}}$ & $3.86 \pm 0.12$ & $7.03 \pm 0.08$ & $7.47 \pm 0.01$ & $6.56 \pm 0.11$ & $7.14 \pm 0.01$ \\
\hline Acetate $(g / L)^{a}$ & $4.85 \pm 0.14$ & $1.14 \pm 0.01$ & $1.04 \pm 0.01$ & $1.13 \pm 0.01$ & $1.31 \pm 0.09$ \\
\hline Lactate $(\mathrm{g} / \mathrm{L})^{\mathrm{a}}$ & $1.50 \pm 0.11$ & $0.10 \pm 0.01$ & $0 \pm 0$ & $0.07 \pm 0.01$ & $0.06 \pm 0.01$ \\
\hline Succinate $(g / L)^{a}$ & $4.38 \pm 0.36$ & $0 \pm 0$ & $0 \pm 0$ & $0 \pm 0$ & $0 \pm 0$ \\
\hline$D C W(g / L)^{a, b}$ & $3.59 \pm 0.11$ & $5.83 \pm 0.08$ & $7.63 \pm 0.02$ & $7.59 \pm 0.20$ & $7.10 \pm 0.35$ \\
\hline Total $(g / L)^{c}$ & $19.48(15.98)$ & $14.71(8.88)$ & $16.44(8.81)$ & $15.72(8.18)$ & $15.91(8.81)$ \\
\hline
\end{tabular}

${ }^{a}$ Each value was determined after $122 \mathrm{~h}$ of cultivation during 3-amino-4-hydroxybenzoic acid (3,4-AHBA) production by recombinant C. glutamicum strain KT01. Data are presented as the mean \pm standard deviation calculated from the results of duplicate independent experiments. Strain KT01 was grown under aerobic conditions until the late log phase in brain heart infusion medium, and the cultures were then inoculated at an initial $\mathrm{OD}_{600}$ of 0.2 into modified mineral salt CGX II medium containing glucose $(40 \mathrm{~g} / \mathrm{L})$ as the sole carbon source

${ }^{\mathrm{b}}$ Dry cell weight (DCW) was determined by the following equation: an $\mathrm{OD}_{600}$ of 1.0 corresponded to 0.39 mg dry weight cell per milliliter

${ }^{\mathrm{C}}$ Values in parenthesis indicate the total amounts of the produced metabolites 


\section{Results}

\section{Enhanced 3,4-AHBA production under low} DO concentration

To examine the effect of DO on 3,4-AHBA production from glucose by recombinant C. glutamicum, the DOstat method was implemented to control DO levels in culture medium at 1.3, 2.6, 5.3, and 8.0 ppm (representing the range from 17 to $100 \%$ saturated oxygen concentration) after $24 \mathrm{~h}$ of cultivation. In culture at a DO level of $0 \mathrm{ppm}$ (representing $0 \%$ saturated oxygen concentration), the agitation speed was fixed at $200 \mathrm{rpm}$ to ensure that the oxygen supply was the rate-limiting step for C. glutamicum cells to produce 3,4-AHBA. After $72 \mathrm{~h}$ of cultivation, the specific productivity of 3,4-AHBA showed a negative correlation with the DO level (Fig. 2). The specific productivity of 3,4-AHBA was comparable $(0.4 \mathrm{mg} 3,4-\mathrm{AHBA} / \mathrm{h} / \mathrm{g}$ dry cell weight $[\mathrm{DCW}])$ at high DO levels $(\geq 2.6 \mathrm{ppm})$, while it sharply increased with reduced DO levels $\leq 1.3 \mathrm{ppm}$, reaching $3.5 \mathrm{mg} / \mathrm{h} / \mathrm{g}$ DCW at a DO level of $0 \mathrm{ppm}$.

Compared to a high DO level (2.6 ppm), cell growth was reduced by $44 \%$ after $72 \mathrm{~h}$ of cultivation under a low DO level of 0 ppm, whereas the glucose consumption rate was comparable throughout the period of $122 \mathrm{~h}$ of cultivation (Fig. 3). Conversely, 3,4-AHBA concentration after $122 \mathrm{~h}$ of cultivation was 4.4-fold higher at a DO level of $0 \mathrm{ppm}$ than at $2.6 \mathrm{ppm}$. These results suggest that oxygen limitation enhances 3,4-AHBA production, probably by directing more carbon and nitrogen from cell growth to 3,4-AHBA production.

\section{Metabolic shift from amino acids to organic acids under low DO conditions}

To investigate enhanced 3,4-AHBA production under oxygen limitation, extracellular concentrations of amino acids and organic acids during 3,4-AHBA production were determined. At DO levels $\geq 1.3 \mathrm{ppm}$, the composition and concentration of external amino acids were comparable (Fig. 4). After $122 \mathrm{~h}$ of cultivation, the total amino acid content was approximately $8.5 \mathrm{~g} / \mathrm{L}$, with Lys predominating (from 6.8 to $7.2 \mathrm{~g} / \mathrm{L}$ ), followed by glycine $(\mathrm{Gly} \leq 0.2 \mathrm{~g} / \mathrm{L})$. In contrast, at the low DO level of $0 \mathrm{ppm}$, the concentration of total amino acid reduced to $3.9 \mathrm{~g} / \mathrm{L}$ (Table 1), with alanine (Ala) predominating, followed by Lys and valine (Val) $(1.5,1.3$, and $0.9 \mathrm{~g} / \mathrm{L}$, respectively). Under oxygen limitation, a significant amount of organic acid was accumulated. After $122 \mathrm{~h}$ of cultivation, the concentrations of succinate, acetate, and lactate were 4.4, 4.9, and $1.5 \mathrm{~g} / \mathrm{L}$, respectively (Fig. 4). In contrast, at DO levels $\geq 1.3 \mathrm{ppm}$, the concentration of acetate was reduced by $73 \%(\leq 1.3 \mathrm{~g} / \mathrm{L})$, and succinate and lactate were scarcely observed. The total amount of metabolites produced was comparable in the range of $8.18-8.88 \mathrm{~g} / \mathrm{L}$ at DO levels $\geq 1.3 \mathrm{ppm}$, while the total amount increased by about two-fold $(15.98 \mathrm{~g} / \mathrm{L})$ at $\mathrm{DO}=1 \mathrm{ppm}$ (Table 1$)$. These results suggest that glucose metabolism drastically shifted at DO levels $<1.3 \mathrm{ppm}$ to produce organic acids instead of amino acids, and that reduced formation of Lys can increase the usage of carbon available for 3,4-AHBA formation.

\section{Metabolic profiles at three different DO concentrations}

To investigate the change in the metabolic state under oxygen limitation, the metabolic profiles of C. glutamicum cells during 3,4-AHBA production were compared at three different DO levels $(0,2.6$, and $5.3 \mathrm{ppm})$. In the central metabolic pathway, most metabolic intermediates showed similar profiles at DO levels of 2.6 and $5.3 \mathrm{ppm}$, while some exhibited specific profiles under oxygen limitation $(\mathrm{DO}=0 \mathrm{ppm})$ (Fig. 5a). For instance, in the glycolytic pathway, reduced levels of fructose-6-phosphate and significantly increased levels of fructose-1,6-bisphosphate (FBP), glyceraldehyde-3-phosphate (GAP), and pyruvate were observed at a DO level of $0 \mathrm{ppm}$, as compared to $\mathrm{DO} \geq 2.6 \mathrm{ppm}$ (Fig. 5a). In addition, the level of DHAP, a precursor for 3,4-AHBA synthesis, was markedly increased under oxygen limitation. Acetyl-CoA (AcCoA) levels were reduced with decreasing DO levels, while significantly increased levels of pyruvate, and its derivative of lactate, were observed under oxygen limitation ( $\mathrm{DO}=0 \mathrm{ppm})$. In the TCA cycle, levels of five sequential metabolic intermediates (iso-citrate, 2-oxoglutarate, succinate, fumarate, and malate) were significantly increased under oxygen limitation ( $\mathrm{DO}=0 \mathrm{ppm})$. In oxidative PPP, the levels of most metabolic intermediates gradually reduced with time, and the level of 6-phosphoD-glucono-1,5-lactone (6PG) was significantly reduced at lower DO levels (Fig. 5a). In cofactor metabolism, levels of both $\mathrm{NAD}^{+}$and $\mathrm{NADP}^{+}$were significantly lower under oxygen limitation $(\mathrm{DO}=0 \mathrm{ppm})$ compared to those at DO levels $\geq 2.6 \mathrm{ppm}$. Conversely, the levels of adenosine 5'-triphosphate (ATP) and adenosine 5 '-diphosphate (ADP) were not significantly different.

In contrast to the central metabolic pathway, DO levels had limited effects on the intracellular levels of amino acids (Fig. 5b). However, markedly reduced levels of glutamine were observed, whereas glutamate levels were relatively high at a DO of $0 \mathrm{ppm}$, resulting in a significantly increased ratio of glutamate/glutamine under oxygen limitation, as compared to DO levels $\geq 2.6 \mathrm{ppm}$. Under oxygen limitation $(\mathrm{DO}=0 \mathrm{ppm})$, significantly increased levels of alanine and lactate, both pyruvate derivatives, were observed. For amino acids derived from aspartate (Asp), which serves as a precursor for 3,4-AHBA synthesis, levels of Asp were increased markedly and those of 


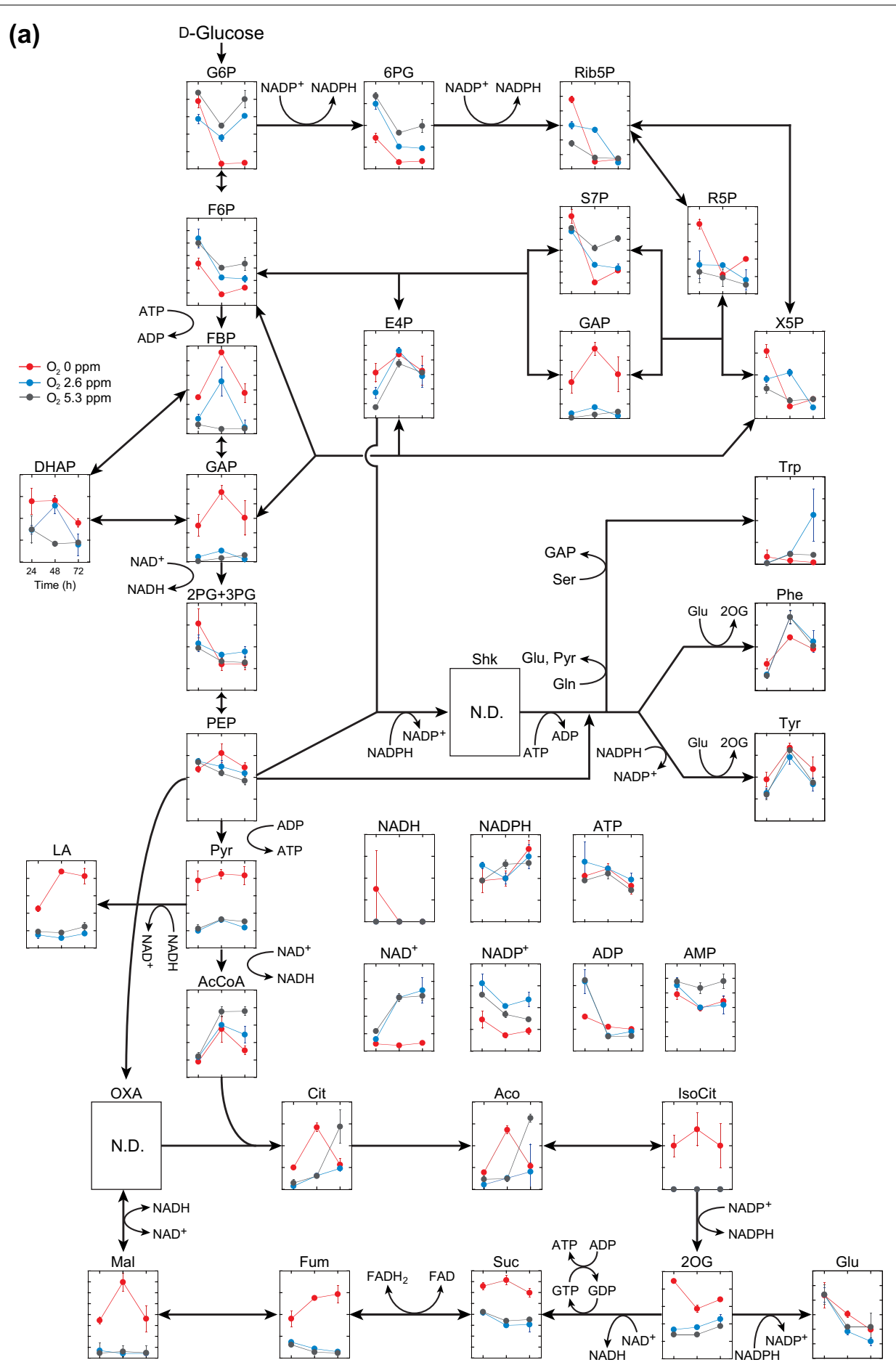

Fig. 5 Time course metabolomics of in the central metabolic pathway (a) and amino acid metabolism (b). Relative abundances of metabolic intermediates in recombinant C. glutamicum strain KT01 during 3-amino-4-hydroxybenzoic acid (3,4-AHBA) production under controlled dissolved oxygen (DO) levels at 0, 2.6, and 5.3 ppm using the DO-stat program in synthetic CGX II medium containing D-glucose as the sole carbon source are shown. The X-axis indicates cultivation time (h). For the profiling experiment, strain KT01 was grown under aerobic conditions until the late log phase in brain heart infusion medium, and the culture was then inoculated to an initial OD600 of 0.2 into CGX II medium containing D-glucose as the sole carbon source ( $40 \mathrm{~g} / \mathrm{L}$ ). The cells were harvested at 24,48 , and $72 \mathrm{~h}$ of cultivation and subsequently subjected to metabolome analysis. Data are presented as average \pm standard deviation calculated from the results of triplicate individual experiments. N.D. not determined below the detection limit 


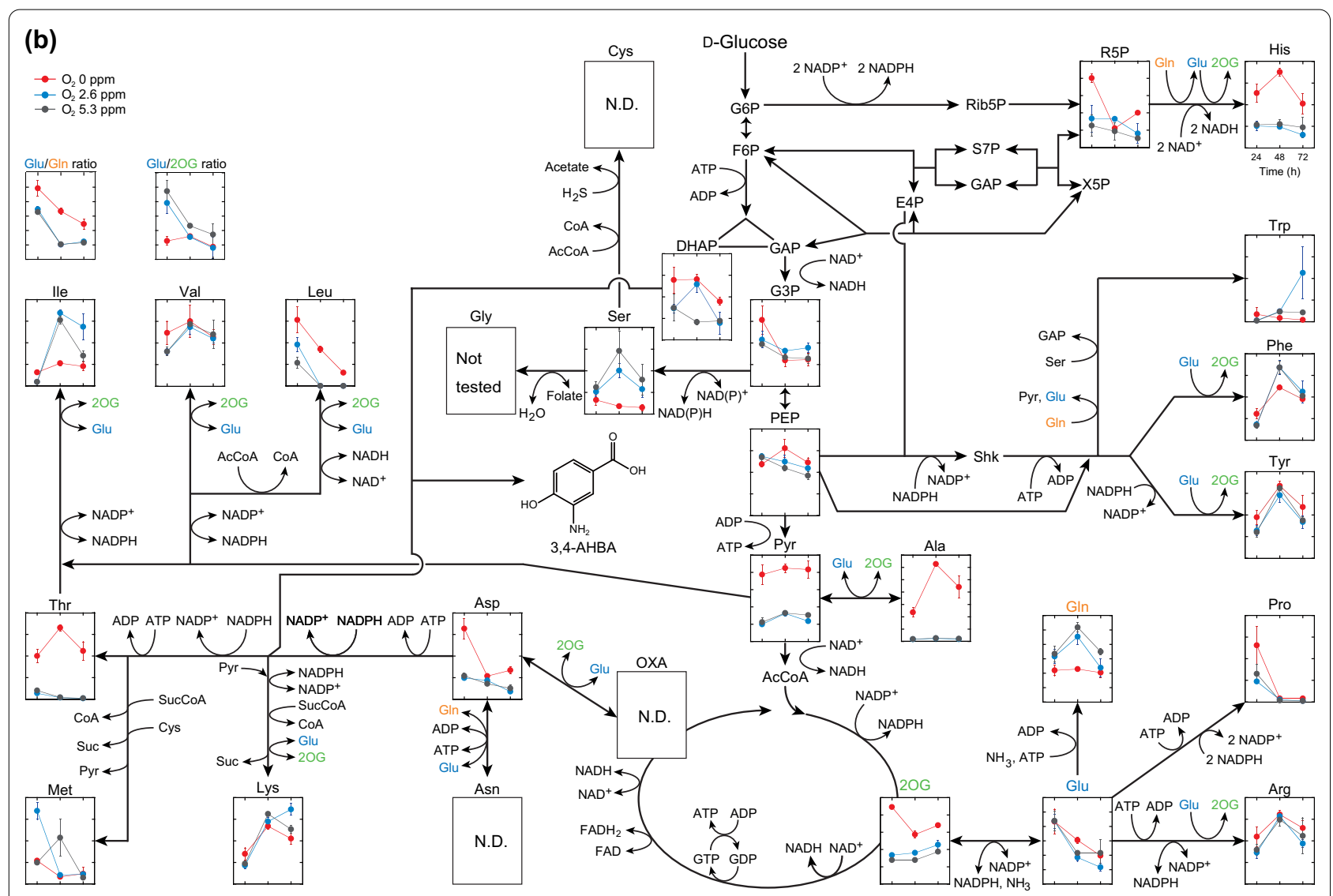

Fig. 5 continued

threonine were significantly increased at $\mathrm{DO}=0 \mathrm{ppm}$, while Lys levels were comparable at the three DO levels (Fig. 5b). Under all conditions, leucine levels monotonically decreased as time progressed due to the auxotrophy for leucine of the host strain ATCC 21799 [24]. These results suggest that glucose metabolism, including amino acid synthesis, drastically changes under oxygen limitation at DO levels $<2.6 \mathrm{ppm}$ during 3,4-AHBA production.

\section{Metabolic engineering for enhanced 3,4-AHBA production}

The host strain ATCC 21799 was metabolically engineered to design a metabolic pathway tailored for oxygen limitation during 3,4-AHBA production. To use excess pyruvate under oxygen limitation for 3,4-AHBA production, four biosynthetic pathways of by-products were selected for analysis based on metabolome data (Fig. 5). To eliminate or reduce by-products of acetate, lactate, succinate, or alanine, the following were individually or collectively inactivated in the wild-type strain by gene disruption (Fig. 1): pyruvate dehydrogenase (encoded by pqo), succinyl-CoA:acetate CoA-transferase (encoded by cat), phosphate acetyltransferase (encoded by pta), and acetate kinase (encoded by ack $A$ ) for acetate synthesis, lactate dehydrogenase (encoded by $l d h$ ) for lactate synthesis, malate dehydrogenase (encoded by $m d h$ ) for succinate synthesis, and two aminotransferases (encoded by alaT and $a v t A$ ) for alanine synthesis. A single $\Delta l d h$ mutant (HKC5021) and a double $\Delta a l a T \Delta a v t A$ mutant (HKC5044) were developed to inactivate the formation of lactate and alanine, respectively (Table 2). To reduce the formation of acetate and succinate, a triple $\Delta p q o \Delta c a t \Delta p t a-a c k A$ mutant (HKC5050) and single $\Delta m d h$ mutant (HKC5053) were also developed. These mutants were transformed by introducing the plasmid pCACgriHI, and the resulting transformants were selected based on chloramphenicol resistance and 3,4AHBA from glucose (Table 2).

Using metabolically engineered strains of $C$. glutamicum, the specific productivity of 3,4-AHBA was compared to that of the parent strain. Compared to the parent strain KT01, the specific productivity of 3,4AHBA was more than double in the $\Delta l d h$ (HKC5037) and $\Delta p q o \Delta c a t \Delta p t a-a c k A$ (HKC5052) mutants, whereas 
Table 2 Strains and plasmids used in this study

\begin{tabular}{|c|c|c|}
\hline Name & Relevant characteristics & Reference or source \\
\hline \multicolumn{3}{|l|}{ Strain } \\
\hline Escherichia coli JM109 & 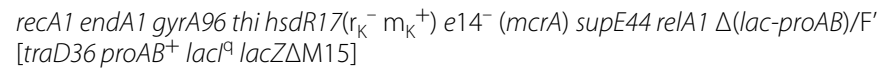 & Takara Bio \\
\hline Corynebacterium glutamicum ATCC 21799 & L-Lysine producer resistant to S-2-aminoethyl-L-cysteine, a lysine analog & ATCC \\
\hline C. glutamicum strain KT01 & C. glutamicum ATCC 21799 bearing pCACgriHI & Kawaguchi et al. [11] \\
\hline C. glutamicum strain HKC5021 & Markerless mutant, $\Delta / d h$ (KaCgl_14550) of strain ATCC 21799 & This study \\
\hline C. glutamicum strain HKC5044 & $\begin{array}{l}\text { Markerless double mutant, } \triangle a l a T \triangle \triangle a v t A \text { (KaCgl_13940 and KaCgl_11440, } \\
\text { respectively) of strain ATCC } 21799\end{array}$ & This study \\
\hline C. glutamicum strain HKC5050 & 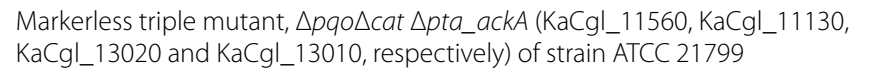 & This study \\
\hline C. glutamicum strain HKC5053 & Markerless mutant of $\Delta m d h$ (KaCgl_08800) of strain ATCC 21799 & This study \\
\hline C. glutamicum strain HKC5037 & HKC5021 bearing pCACgriHI & This study \\
\hline C. glutamicum strain HKC5051 & HKC5044 bearing pCACgriHI & This study \\
\hline C. glutamicum strain HKC5052 & HKC5050 bearing pCACgriHI & This study \\
\hline C. glutamicum strain HKC5057 & HKC5053 bearing pCACgriHI & This study \\
\hline \multicolumn{3}{|l|}{ Plasmid } \\
\hline pCACgriHl & $\begin{array}{l}\mathrm{Cm}^{\mathrm{r}} \text {; E. coli-Corynebacterium sp. shuttle vector harboring griH and gril genes } \\
\text { derived from Streptomyces griseus for 3,4-AHBA biosynthesis }\end{array}$ & Kawaguchi et al. [11] \\
\hline pK19mobsacB & $\begin{array}{l}\text { Kan', mobilizable E. coli vector for the construction of insertion and deletion } \\
\text { mutants of C. glutamicum (oriV, sacB, lacZa) }\end{array}$ & ATCC \\
\hline pK19mobsac- $\Delta$ pta-ackAldh & $\begin{array}{l}\text { Kan', pK19mobsacB with the deletion construct for pta-ackA (KaCgl_13010 } \\
\text { and KaCgl_13020) of C.glutamicum ATCC } 21799\end{array}$ & This study \\
\hline pK19mobsac- $\triangle a l a T$ & $\begin{array}{l}\text { Kan', pK19mobsacB with the deletion construct for alaT (KaCgl_13940) of C. } \\
\text { glutamicum ATCC } 21799\end{array}$ & This study \\
\hline pK19mobsac- $\triangle a v t A$ & $\begin{array}{l}\text { Kan', pK19mobsacB with the deletion construct for avtA (KaCgl_11440) of C. } \\
\text { glutamicum ATCC } 21799\end{array}$ & This study \\
\hline pK19mobsac- $\Delta$ cat & $\begin{array}{l}\text { Kan', pK19mobsacB with the deletion construct for cat (KaCgl_11130) of C. } \\
\text { glutamicum ATCC } 21799\end{array}$ & This study \\
\hline pK19mobsac- $\Delta / d h$ & $\begin{array}{l}\text { Kan', pK19mobsacB with the deletion construct for Idh (KaCgl_14550) of C. } \\
\text { glutamicum ATCC } 21799\end{array}$ & This study \\
\hline pK19mobsac- $\Delta m d h$ & $\begin{array}{l}\text { Kan', pK19mobsacB with the deletion construct for mdh (KaCgl_08800) of C. } \\
\text { glutamicum ATCC } 21799\end{array}$ & This study \\
\hline pK19mobsac- $\triangle p q 0$ & $\begin{array}{l}\text { Kan', pK19mobsacB with the deletion construct for pqo (KaCgl_29450) of C. } \\
\text { glutamicum ATCC } 21799\end{array}$ & This study \\
\hline
\end{tabular}

the cell growth of these strains was significantly reduced (Table 3). A double $\Delta a l a T \Delta a v t A$ mutant (HKC5051), which had the auxotrophy for alanine, showed a 1.2-fold higher specific productivity of 3,4-AHBA than the parent strain with reduced cell growth. In contrast, the specific productivity of 3,4-AHBA was reduced by $90 \%$ in the $\Delta m d h$ mutant (HKC5057), with significantly reduced cell growth, as compared to the parent strain KT01. During 3,4-AHBA production, the total amount of by-products formed was significantly lower in all of the metabolically engineered strains than in the parent strain KT01; in particular, the accumulation of organic acids (acetate, lactate, and succinate) was significantly reduced (Table 3). In addition, Lys formation was comparable in $\Delta l d h$ (HKC5037) but significantly increased in the remaining three mutants, $\Delta p q o \Delta c a t \Delta p t a-a c k A$ (HKC5052),
$\Delta a l a T \Delta a v t A$ (HKC5051), and $\Delta m d h$ (HKC5057), as compared to that in the parent strain.

\section{Improved 3,4-AHBA production by a rationally designed strain of $C$. glutamicum}

Based on the comparative results shown in Table 3, the $\Delta l d h$ mutant (HKC5037) was selected for improved 3,4-AHBA production with the highest specific productivity, and the 3,4-AHBA production was compared with the parent strain KT01 in glucose fed-batch fermentation. The fed-batch fermentation was conducted with constant agitation at $200 \mathrm{rpm}$ to maintain the DO level at $0 \mathrm{ppm}$ after $24 \mathrm{~h}$ of cultivation. After $228 \mathrm{~h}$ of cultivation, $5.6 \mathrm{~g} / \mathrm{L}$ of $3,4-\mathrm{AHBA}$ was produced by the metabolically engineered $C$. glutamicum HKC5037 strain ( $\Delta l d h$ mutant), whereas the parent 
Table 3 3-amino-4-hydroxybenzoic acid (3,4-AHBA) production from glucose by metabolically engineered strains of C. glutamicum

\begin{tabular}{|c|c|c|c|c|c|}
\hline & $\begin{array}{l}\text { Parent } \\
\text { (KT01) }\end{array}$ & $\begin{array}{l}\Delta l d h \\
\text { (HKC5037) }\end{array}$ & $\begin{array}{l}\Delta p q o \Delta c a t \Delta p t a-a c k A \\
\text { (HKC5052) }\end{array}$ & $\begin{array}{l}\text { DalaTAavt } A^{\mathrm{c}} \\
\text { (HKC5051) }\end{array}$ & $\begin{array}{l}\Delta m d h \\
\text { (HKC5057) }\end{array}$ \\
\hline 3,4-AHBA (g/L) ${ }^{a}$ & $1.140 \pm 0.085$ & $1.950 \pm 0.283$ & $0.906 \pm 0.199$ & $1.190 \pm 0.046$ & $0.030 \pm 0.017$ \\
\hline Cell growth (g DCW/L) ${ }^{\mathrm{a}}$ & $7.2 \pm 0.3$ & $3.5 \pm 0.5$ & $2.9 \pm 0.6$ & $6.6 \pm 0.9$ & $2.8 \pm 1.1$ \\
\hline $\begin{array}{l}\text { Specific productivity } \\
(\mathrm{mg} / \mathrm{h} / \mathrm{g} \mathrm{DCW})^{\mathrm{a}}\end{array}$ & $157.9 \pm 5.7$ & $561.7 \pm 1.2$ & $314.8 \pm 31.5$ & $183.3 \pm 24.0$ & $14.6 \pm 15.1$ \\
\hline Relative productivity $(\%)^{\mathrm{b}}$ & $100 \pm 4$ & $365 \pm 1$ & $205 \pm 20$ & $119 \pm 16$ & $10 \pm 10$ \\
\hline \multicolumn{6}{|l|}{ By-product } \\
\hline Lactate $(g / L)^{a}$ & $1.12 \pm 0.35$ & N.D. & N.D. & $0.54 \pm 0.10$ & $0.02 \pm 0.01$ \\
\hline Acetate $(g / L)^{a}$ & $2.88 \pm 0.81$ & $0.90 \pm 0.49$ & $0.81 \pm 0.01$ & $1.63 \pm 0.22$ & N.D. \\
\hline Succinate $(g / L)^{a}$ & $3.39 \pm 0.28$ & N.D. & N.D. & $0.39 \pm 0.39$ & N.D. \\
\hline Ala $(g / L)^{a}$ & $1.51 \pm 0.07$ & $0.54 \pm 0.02$ & $0.63 \pm 0.02$ & $0.21 \pm 0.04$ & N.D. \\
\hline $\operatorname{Val}(g / L)^{a}$ & $0.92 \pm 0.01$ & $0.69 \pm 0.07$ & $0.66 \pm 0.00$ & $0.60 \pm 0.11$ & N.D. \\
\hline Lys $(g / L)^{a}$ & $1.30 \pm 0.06$ & $1.60 \pm 0.18$ & $2.86 \pm 0.07$ & $4.06 \pm 0.08$ & $2.55 \pm 0.49$ \\
\hline Total amounts of by-product (g/L) & 11.12 & 3.73 & 4.96 & 7.43 & 2.57 \\
\hline
\end{tabular}

N.D. not detected

${ }^{a}$ Values were determined after $100 \mathrm{~h}$ of cultivation. Data are presented as the mean \pm standard deviation calculated from the results of duplicate independent experiments. Specific productivity was determined based on dry cell weight (DCW) after $100 \mathrm{~h}$ of cultivation. All strains were grown under aerobic conditions until the late log phase in brain heart infusion medium, and the cultures were then inoculated at an initial $\mathrm{OD}_{600}$ of 0.2 into modified mineral salt CGX II medium containing glucose $(40 \mathrm{~g} / \mathrm{L})$ as the sole carbon source

${ }^{\mathrm{b}}$ Relative productivity was determined based on the specific productivity of 3,4-AHBA

CFor 3,4-AHBA production, alanine (final $100 \mathrm{mg} / \mathrm{L}$ ) was added to CGX II medium because this mutant required alanine for cell growth

strain KT01 produced 4.2 g/L of 3,4-AHBA (Fig. 6a, b). Within the first feed of glucose (75 h of cultivation), the glucose consumption rate and cell growth of the HKC5037 strain were comparable with that of the parent strain. This was attained by including urea as the nitrogen source in the CGXII medium to improve the poor growth of the HKC5037 strain under oxygen limitation, as shown in Table 3 and Additional file 1: Fig. S1. The ldh HKC5037 mutant completely lost its ability to produce lactate, while the transient accumulation of succinate after $168 \mathrm{~h}$ of cultivation increased by more than two folds than that in the parent strain (10.8 and $5.1 \mathrm{~g} / \mathrm{L}$, respectively) (Fig. 6c, d). In addition, the acetate concentration after $216 \mathrm{~h}$ of cultivation was 1.5-fold higher in the culture with the HKC5037 strain than the parent strain (8.7 and $5.8 \mathrm{~g} / \mathrm{L}$, respectively), although the production rate was comparable. Consequently, 3,4-AHBA yield after $288 \mathrm{~h}$ of cultivation was 1.4-fold higher by the rationally designed strain HKC5037 than that in the parent strain (0.070 and $0.053 \mathrm{~g}$ of 3,4-AHBA/g of glucose, respectively).

\section{Discussion}

The pathway of 3,4-AHBA synthesis is specific to the actinomycete Streptomyces griseus. In this strain, the aromatic ring is formed through a two-step reaction from primary metabolites of DHAP in glycolysis and L-aspartate-4-semialdehyde as the Asp metabolite by the aldol condensation reaction [10], whereas most aromatic compounds in bacteria are predominantly synthesized in a multistep reaction via the shikimate pathway, which is subjected to multiple feedback regulations $[1,9,25]$. $C$. glutamicum strain ATCC 21799 has a gene for a feedback-resistant aspartate kinase in its chromosome [26], and produces a large amount of Lys under aerobic conditions (50\% DO saturation) [27]. In contrast, the present study demonstrated that the carbon flux of L-aspartate4-semialdehyde shifted toward 3,4-AHBA instead of Lys under oxygen limitation at a DO level of $0 \%$.

Oxygen limitation changes the redox state of microbial cells $[12,28]$. For the production of aromatic compounds, the DO level is generally controlled at $\geq 20 \%$ of saturated oxygen, such as the production of phenylalanine (Phe) [29] by C. glutamicum and the production of tryptophan (Trp) [30], shikimate [19], and Tyr [31] by E. coli. In contrast, the specific productivity of 3,4-AHBA increased with reduced DO levels, reaching a maximum concentration at a DO concentration of 0 ppm (Fig. 2). Apparently, glucose metabolism was shifted at a threshold of DO level between 0 and $1.3 \mathrm{ppm}$ (corresponding to $17 \% \mathrm{O}_{2}$ saturation). Consequently, the concentration of Lys, a predominant amino acid, was reduced by $81 \%$, and the amount of organic acids conversely increased 8.3-fold under $\mathrm{DO}=0 \mathrm{ppm}$, as compared to the aerobic conditions (Fig. 4). In contrast, the composition and concentration of extracellular amino acids and organic acids were comparable at DO levels $\geq 1.3 \mathrm{ppm}$. Even at a DO concentration of $1.3 \mathrm{ppm}$, the specific productivity of 3,4-AHBA 

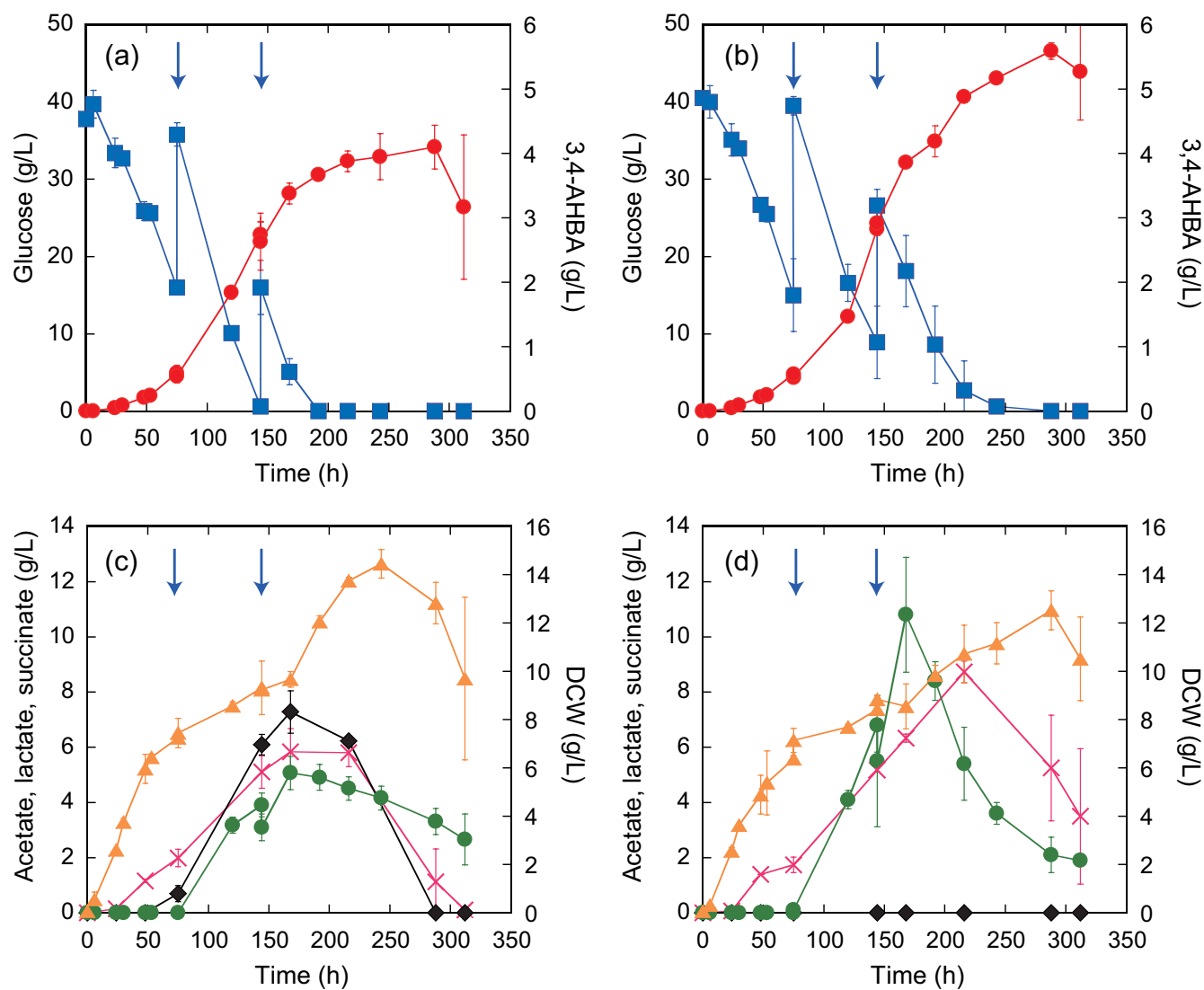

Fig. 6 3-Amino-4-hydroxybenzoic acid (3,4-AHBA) production in glucose fed-batch cultures using two metabolically engineered C. glutamicum strains. For the profiling experiment, either parent strain KT01 (a and $\mathbf{c}$ ) or strain HKC5037 ( $\Delta /$ dh mutant) (b and $\mathbf{d}$ ) was grown under aerobic conditions until the late log phase in brain heart infusion medium, and the culture was then inoculated to an initial $\mathrm{OD}_{600}$ of 0.2 into $\mathrm{CGX}_{\mathrm{II}}$ medium containing glucose ( $40 \mathrm{~g} / \mathrm{L})$ as the sole carbon sources. Glucose was fed twice as indicated in arrows to increase glucose concentration of $20 \mathrm{~g} / \mathrm{L}$. The concentrations of 3,4-AHBA (red circles), glucose (squares), acetate (crosses), lactate (diamonds), succinate (green circles), and cells (triangles) are shown. Data are presented as average \pm standard deviation calculated from the results of duplicate individual experiments

was reduced by $49 \%$ as compared to $\mathrm{DO}=0 \mathrm{ppm}$ (Fig. 2). Compared to 3,4-AHBA biosynthesis, Lys biosynthesis requires more NADPH (4 mol of NADPH for $1 \mathrm{~mol}$ of Lys) [16], which is predominantly supplied from the oxidative PPP by aerobic metabolism [15, 20, 32] (Fig. 1).

In the present study, intracellular levels of both 6PG in PPP and NADP ${ }^{+}$, as the cofactor for Lys biosynthesis, were significantly lower at a DO level of 0 ppm than at $\geq 2.6 \mathrm{ppm}$ (Fig. 5a). The reduced 6PG level under oxygen limitation conditions implies reduced flux through the oxidative PPP at a DO level of $0 \mathrm{ppm}$. This is supported by a previous study that showed that the flux from glycolysis to PPP decreased with reduced DO levels [33]. However, the NADPH levels were comparable between high and low DO levels, while the $\mathrm{NADP}^{+}$levels were reduced under low DO levels (Fig. 5a). In C. glutamicum, malic enzyme, which reduces $\mathrm{NADP}^{+}$to NADPH coupled with the oxidative decarboxylation of malate to pyruvate (Fig. 1), was induced under oxygen limitation [23]. The enhanced malic enzyme activity can compensate NADPH supply for reduced PPP flux under oxygen limitation [34]. Nevertheless, NADPH supply under oxygen limitation was likely to be limited for amino acid biosynthesis (Table 1), which requires NADPH as a cofactor [15] (Fig. 1). In addition, oxygen limitation induces the expression of genes responsible for organic acid production, which in turn oxidizes NADH to regenerate $\mathrm{NAD}^{+}$, instead of aerobic respiration, for ATP generation $[22,23]$. However, $\mathrm{NAD}^{+}$generation was also limited under oxygen limitation (Fig. 5a), resulting in reduced cell growth (Table 1). Consequently, oxygen limitation $(\mathrm{DO}=0 \mathrm{ppm})$ increased the total amounts of metabolites produced while reducing cell growth and shifted glucose metabolism to produce organic acids instead of amino acids, as compared to $\mathrm{DO} \geq 1.3 \mathrm{ppm}$ (Table 1). These results suggest that a strict rate-limiting oxygen supply 
is critical for the enhanced production of 3,4-AHBA by recombinant $C$. glutamicum to control the redox state.

In addition to the enhanced 3,4-AHBA metabolism, oxygen limitation has another advantage for increased 3,4-AHBA titer. A previous study reported that 3,4AHBA was non-enzymatically oxidized to form a yellow pigment under aerobic conditions [10]. This suggests that oxygen limitation $(\mathrm{DO}=0 \mathrm{ppm})$ can attenuate the oxidation of the 3,4-AHBA produced, occurring under aerobic conditions ( $\mathrm{DO} \geqq 2.6 \mathrm{ppm}$ ). To the best of our knowledge, this is the first report on the enhanced production of aromatic compounds under strictly oxygen-limited conditions at a DO concentration of $0 \mathrm{ppm}$.

Controlling the oxygen supply and thereby recycling cofactor $\operatorname{NAD}(\mathrm{P})^{+}$is one strategy to optimize the performance of fermentation. However, knowledge regarding the comparative profiles between aerobic and oxygenlimited conditions remains limited [12]. Comparable metabolome analysis would provide insights into the change in metabolic status in response to DO concentration in both sugar and amino acid metabolism. Besides 3,4-AHBA production, metabolic profiles were altered at a DO threshold level between $0 \mathrm{ppm}$ and $2.6 \mathrm{ppm}$. At a DO level of $0 \mathrm{ppm}$, intracellular concentrations of NAD ${ }^{+}$ and $\mathrm{NADP}^{+}$were significantly lower than those under aerobic conditions (Fig. 5a). Under oxygen limitation, the intracellular concentration of $\mathrm{NAD}^{+}$was significantly reduced due to the shortage of $\mathrm{NAD}^{+}$recycling coupled with aerobic respiration [35], although some $\mathrm{NAD}^{+}$can be regenerated by lactate dehydrogenase and malate dehydrogenase under oxygen limitation [36]. Moreover, the limited $\mathrm{NAD}^{+}$recycling blocked all redox reactions requiring $\mathrm{NAD}^{+}$as a cofactor and consequently accumulated pyruvate and GAP (Fig. 5a). At a DO level of 0 ppm, a reduced $\mathrm{NAD}^{+} / \mathrm{NADH}$ ratio, associated with the downregulation of the NADH dehydrogenase gene, was also observed in C. glutamicum cells in a previous study [21].

The accumulation of pyruvate resulted in increased intracellular and extracellular lactate levels and alanine at a DO level of 0 ppm (Figs. 4, 5a, b). Under oxygen limitations, intracellular levels of Asp was markedly increased, and that of threonine (Thr) was significantly increased, while Lys levels were unaffected (Fig. 5b), suggesting that the synthesis of amino acids, including Lys, is suppressed due to limited NADPH supply [37] and that the surplus L-aspartate-4-semialdehyde can be used for 3,4-AHBA synthesis. Under oxygen limitation, upregulation of triosephosphate isomerase, which catalyzes the reversible conversion of GAP and DHAP, was observed in previous studies [21, 23], which allows an increase in DHAP supply for 3,4-AHBA biosynthesis (Fig. 5a). In previous studies, genome-based flux balance analysis was adopted to predict and measure the operation and regulation of metabolic networks [38]. In contrast, the present metabolomics analysis revealed metabolic shifts in both the central metabolic pathway and amino acid metabolism, particularly in pyruvate-derived amino acids under strict oxygen limitation $(\mathrm{DO}=0 \mathrm{ppm})$, although intracellular concentrations of amino acids were comparable between high and low DO levels [22].

Comparative analysis of both extracellular and intracellular concentrations of metabolites between aerobic and oxygen-limited conditions pointed to a metabolic shift in response to DO concentration (Figs. 4, 5a, b). Based on these results, the metabolic pathway of the 3,4-AHBAproducing strain KT01 was rationally engineered to perform at its full potential under oxygen limitation (Fig. 1, Table 3). The $m d h$ mutant showed poor cell growth and produced negligible levels of 3,4-AHBA under oxygen limitation (Table 3 ). The $m d h$ gene encoding malate dehydrogenase plays an important role in $\mathrm{NAD}^{+}$recycling under anaerobic conditions [36], and the $m d h$ mutant showed a significantly reduced glucose consumption rate [32]. These results suggest that $m d h$ disruption remarkably diminishes the capability of $\mathrm{NAD}^{+}$recycling at a DO concentration of $0 \mathrm{ppm}$. In addition, the inability to convert oxaloacetate (OXA) into malate blocked succinate production, but the excess OXA was likely to be used for enhanced Lys production (Table 3). In C. glutamicum, two aminotransferases encoded by ala $T$ and avt $A$ are exclusively responsible for alanine synthesis [39]. The specific productivity of 3,4-AHBA in the double mutant $\triangle a l a T \Delta a v t A$ was comparable to that of the parent strain (Table 3). If the alanine biosynthetic pathway is blocked, the precursors of pyruvate and glutamate can be used for the synthesis of other pyruvate-derived metabolites [40]. Consequently, this mutant showed the highest productivity for total by-products, especially Lys, among the four metabolically engineered strains (Fig. 4), suggesting a limited effect of the $\triangle$ alaT $\Delta a v t A$ mutant on enhanced 3,4-AHBA production. The $\Delta l d h$ mutant and $\Delta p q o \Delta c a t \Delta p t a$-ackA triple mutant were designed to lose lactate productivity [41] or reduce acetate productivity [42], respectively. These two strains showed more than two times increased specific productivity of 3,4-AHBA compared to that of the parent strain KT01 (Table 3). Compared to the $\Delta l d h$ mutant, the $\Delta p q o \Delta c a t \Delta p t a-a c k A$ triple mutant showed 1.8-fold higher Lys productivity, while other metabolites were produced at comparable levels. In contrast, the $\Delta l d h$ mutant showed the lowest productivity of Lys, while the levels of other pyruvatederived metabolites, such as acetate, Ala, and Val, were comparable to that of the $\Delta p q o \Delta c a t \Delta p t a-a c k A$ triple mutant. These results suggest that the lower production of other by-products allows the $\Delta l d h$ mutant to improve its specific productivity of 3,4-AHBA. 


\section{Conclusions}

In the present study, we demonstrated that both extracellular and intracellular metabolic profiles were constant at $\mathrm{DO} \geq 2.6 \mathrm{ppm}$, corresponding to $\geq 33 \%$ saturated oxygen at $26^{\circ} \mathrm{C}$; however, this profile changed remarkably under oxygen limitation $(\mathrm{DO}=0 \mathrm{ppm})$. Consequently, we revealed that 3,4-AHBA production reached the maximum level at a DO of $0 \mathrm{ppm}$, whereas the fermentation of other aromatic compounds was optimized at a DO of $\geq 10 \%$ saturated oxygen. To the best of our knowledge, this is the first study to report a comparative measurement of metabolic intermediates instead of a conventional approach using genome-based flux balance analysis to optimize DO conditions for microbial production. The metabolomics analysis revealed the points at which the metabolism shifted in response to DO concentration, particularly under oxygen limitation $(\mathrm{DO}=0 \mathrm{ppm})$. Under oxygen limitation, we observed significantly reduced $\operatorname{NAD}(\mathrm{P})^{+}$levels and altered levels of relevant metabolic intermediates requiring $\mathrm{NAD}(\mathrm{P})^{+}$as cofactors. These comprehensive data regarding metabolic states in response to DO provide insights into the oxygen supply during fermentation and the rational design of metabolic pathways for improved production of related amino acids and their derivatives.

\section{Methods}

\section{Bacterial strains and media}

The bacterial strains and plasmids used in this study are listed in Table 2. For genetic manipulations, E. coli strains were grown at $37{ }^{\circ} \mathrm{C}$ in Luria-Bertani medium [43]. For the aerobic growth of C. glutamicum, the wildtype and recombinant strains were grown at $30{ }^{\circ} \mathrm{C}$ to the late log phase in brain heart infusion (BHI) broth (BD Biosciences, Franklin Lakes, NJ, USA), unless indicated otherwise. For AHBA production, modified mineral salt CGXII medium [44] containing glucose as the carbon

Table 4 Oligonucleotides used in this study

\begin{tabular}{|c|c|c|c|}
\hline Name & Target gene & Sequence $\left(5^{\prime}-3^{\prime}\right)$ & Cohesive ends ${ }^{\mathrm{a}}$ \\
\hline Primer 1 & pta-ackA & ACGGCCAGTGGAATTCTGCGTGAGATGAAGTAAGGC & EcoRl \\
\hline Primer 2 & pta-ackA & GGTGTTGGTGAAAATGCCCA & \\
\hline Primer 3 & pta-ackA & ATTTTCACCAACACCACGTGTTTCCTACACCGATG & \\
\hline Primer 4 & pta-ackA & ATGATTACCCAAGCTTGTCCGTGTCGGATTTCATCA & HindIII \\
\hline Primer 5 & alat & ACGGCCAGTGGAATTCCTAGTCCGTTTTCGACGATG & EcoRI \\
\hline Primer 6 & alat & ACAGCACGTCCTTCATCTTC & \\
\hline Primer 7 & alat & TGAAGGACGTGCTGTACCCCAACGTGTACGAAATC & \\
\hline Primer 8 & alat & ATGATTACCCAAGCTTAAGTTTCAGGCATAGGCAGG & HindIII \\
\hline Primer 9 & avtA & ACGGCCAGTGGAATTCTCCATGAGGTCAAGCATGTC & EcoRl \\
\hline Primer 10 & avtA & CCGATGATTCAGAGGAATGG & \\
\hline Primer 11 & avtA & CCTCTGAATCATCGGAGAGCGATCTCTGCTTCTTC & \\
\hline Primer 12 & avtA & ATGATTACCCAAGCTTTTGATGGGGAGACTGTGGTT & HindIII \\
\hline Primer 13 & cat & ACGGCCAGTGGAATTCCGTAAAGCGGAGTTTTAGGC & EcoRI \\
\hline Primer 14 & cat & ATCTCTGAGTACGGTTACGC & \\
\hline Primer 15 & cat & ACCGTACTCAGAGATAACAGGTCGATTGCGTAGTC & \\
\hline Primer 16 & cat & ATGATTACCCAAGCTTAGCAACGTTGGTTACACCAG & HindIII \\
\hline Primer 17 & Idh & ACGGCCAGTGGAATTCTGGGTTAATTCGCCGGTGATCAG & EcoRl \\
\hline Primer 18 & Idh & GGTTGATCAGTGCAGTATGCGTATG & \\
\hline Primer 19 & Idh & CTGCACTGATCAACCCACTGCTCCACGGTGAATACG & \\
\hline Primer 20 & Idh & ATGATTACCCAAGCTTGGCAAGGTCCATGCTGACG & HindIII \\
\hline Primer 21 & $m d h$ & ACGGCCAGTGGAATTCAATGACAACGGCGTGGCTTC & EcoRl \\
\hline Primer 22 & $m d h$ & TCTGCGGCAATTCCTTCCAC & \\
\hline Primer 23 & $m d h$ & AGGAATTGCCGCAGAGGGATCTCCAGAAGTTTCAG & \\
\hline Primer 24 & $m d h$ & ATGATTACCCAAGCTTTTTCCATCAATAGCAGGCGC & HindIII \\
\hline Primer 25 & pqo & ACGGCCAGTGGAATTCTGGTCGCATCTCATCGATTG & EcoRl \\
\hline Primer 26 & pqo & GCATATCCTGGACCTGTACT & \\
\hline Primer 27 & pqo & CAGGATATGCTTTCCAGGACCACAAGAAGC & \\
\hline Primer 28 & pqo & ATGATTACCCAAGCTTCTTGCGCCTGCAAAGTTTCT & HindIII \\
\hline
\end{tabular}

${ }^{a}$ The restriction site overhangs used in the cloning procedure are underlined 
source supplemented with leucine $\left(100 \mathrm{mg} \cdot \mathrm{L}^{-1}\right)$ and pantothenic acid $\left(100 \mathrm{mg} \cdot \mathrm{L}^{-1}\right)$ without urea was used. When appropriate, media were supplemented with $5 \mu \mathrm{g} \cdot \mathrm{mL}^{-1}$ and $50 \mu \mathrm{g} \cdot \mathrm{mL}^{-1}$ chloramphenicol for C. glutamicum and E. coli, respectively.

\section{DNA manipulation}

All restriction endonucleases were purchased from New England Biolabs (Ipswich, MA, USA). PrimeSTAR Max DNA Polymerase (Takara Bio, Kusatsu, Japan) was used for PCR to amplify DNA fragments, according to the manufacturer's instructions. PCR fragments were purified using a QIAquick PCR Purification Kit (Qiagen, Hilden, Germany). Plasmids were developed using an In-Fusion HD Cloning Kit (Takara Bio) for seamless ligation-independent cloning of PCR fragments, according to the manufacturer's instructions. Electroporation was used to transform C. glutamicum, as previously described [45], whereas $E$. coli was transformed using the $\mathrm{CaCl}_{2}$ procedure [43]. Plasmid DNA was isolated from $E$. coli as previously described [46].

\section{Construction of metabolically engineered C. glutamicum strains}

C. glutamicum strain ATCC 21799 was metabolically engineered to improve 3,4-AHBA production based on its whole genome sequence (DDBJ/ENA/GenBank accession number AP022856.1) [47]. Deletion mutants of $C$. glutamicum ATCC 21799 were developed using a suicide vector system followed by a two-step homologous recombination procedure, as previously described [48]. For pta$a c k A$, plasmid pK19mobsac- $\Delta$ ta-ack $A$ was constructed by PCR amplification of the gene with both flanking regions using the C. glutamicum ATCC 21799 genome as the template and oligonucleotide primer pairs 1,2,3, and 4 (Table 4), respectively. The PCR-amplified upstream and downstream fragments were integrated into PstIand EcoRI-digested plasmid pK19mobsacB (American Type Culture Collection, Manassas, VA, USA) by multiple-fragment cloning using In-Fusion, yielding the construct pK19mobsac- $\Delta p t a-a c k A$ (Table 2). Gene deletion was confirmed by PCR using oligonucleotide primers 1 and 4. Analysis of C. glutamicum transformants, selected on the basis of kanamycin sensitivity and sucrose resistance, ultimately yielded a markerless pta-ackA deletion mutant (21799 $\Delta p t a-a c k A)$ (Table 2). Likewise, plasmids pK19mobsac- $\Delta a l a T, \quad$ KK19mobsac- $\Delta a v t A T$, pK19mobsac- $\Delta l d h$, pK19mobsac- $\Delta p q o$, pK19mobsac$\Delta c a t$, and pK19mobsac- $\Delta m d h$ were generated (Table 2), for the transformation of $E$. coli JM109. Using the result-

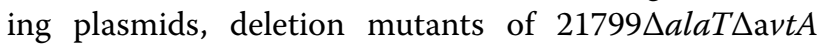
(strain HKC5044), 21799 $\Delta l d h$ (strain HKC5021),

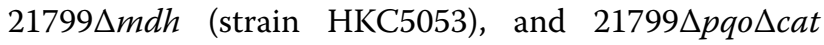

$\triangle p t a-a c k A$ (strain HKC5050) were constructed (Table 2). The deletion mutant was transformed by electroporation with the plasmid pCACgriHI to express griH and griI derived from Streptomyces griseus, which is responsible for 3,4-AHBA synthesis (Table 4) [11]. Transformants were selected by chloramphenicol resistance and were subsequently screened for their ability to produce 3,4AHBA from glucose as the sole carbon source. A positive transformant was selected for further characterization (Table 2).

\section{3,4-AHBA production by recombinant $C$. glutamicum}

Recombinant C. glutamicum strains were grown aerobically to the late log phase in a $50 \mathrm{~mL}$ test tube containing BHI broth with constant agitation (180 rpm) for $18 \mathrm{~h}$ at $26^{\circ} \mathrm{C}$. The pre-culture was transferred to $90 \mathrm{~mL}$ of modified mineral salt CGXII medium containing glucose $\left(40 \mathrm{~g} \mathrm{~L}^{-1}\right.$ glucose $)$ in a $200 \mathrm{~mL}$ jar fermentor Bio Jr.8 BJR-25NA1S-8 M (ABLE Co. \& Biott Co., Tokyo, Japan) to obtain a cell concentration corresponding to an optical density at $600 \mathrm{~nm}\left(\mathrm{OD}_{600}\right)$ of 0.2 . The cultivation conditions were as follows: temperature, $26{ }^{\circ} \mathrm{C} ; \mathrm{pH}, 7.0$, maintained by the addition of ammonia; stirrer speed, $200 \mathrm{rpm}$; aeration with compressed air, $0.5 \mathrm{vvm}$, unless indicated otherwise. For dissolved oxygen (DO)-stat cultivation of 3,4-AHBA production, the DO-stat method was started after $24 \mathrm{~h}$ of cultivation to control DO levels $(0,1.3,2.6,5.3$, and $8.0 \mathrm{ppm})$ by changing the agitation speed.

\section{Analytical procedures}

Culture samples were centrifuged $\left(15,000 \times g, 4{ }^{\circ} \mathrm{C}\right.$, $10 \mathrm{~min}$ ), and the concentrations of 3,4-AHBA, organic acids (acetate, lactate, and succinate), and glucose in the resulting supernatants were measured by high-performance liquid chromatography (HPLC), as previously described [11]. The concentrations of free amino acids in the resulting supernatants were determined with an ultra-high-performance liquid chromatograph (Nexera X2; Shimadzu, Kyoto, Japan) using an AccQ Tag Ultra Chemistry Kit for amino acid analysis (Waters, Milford, MA, USA), as previously described [49]. Cell mass was estimated by measuring the $\mathrm{OD}_{600}$ using a spectrophotometer (U-3010; Hitachi, Tokyo, Japan). An $\mathrm{OD}_{600}$ of 1.0 corresponded to $0.39 \mathrm{mg}$ dry weight cell per milliliter [46].

Metabolome analysis of $C$. glutamicum cells was conducted as previously described [50]. Major metabolites of the central metabolic pathways (e.g., glycolysis, PPP, and tricarboxylic acid [TCA] cycle) were analyzed using an ion-pairing LC-MS/MS method [51]. Dried cell extracts were dissolved in $50 \mu \mathrm{L}$ of MilliQ water for LCMS/MS profiling and quantitation of 30 intracellular $C$. 
glutamicum metabolites. The following metabolites were analyzed: sugar phosphates (glucose-6-phosphate [G6P], fructose-6-phosphate [F6P], frucotose-1,6-bisphosphate [FBP], DHAP, glyceraldehyde-3-phosphate [GAP], 2- and 3-phosphoglycerate [2PG +3PG], phosphoenolpyruvate $[\mathrm{PEP}]$, 6-Phospho-D-glucono-1,5-lactone [6PG], ribulose-5-phosphate [Rib5P], ribose-5-phosphate [R5P], $\mathrm{X} 5 \mathrm{P}$, erythrose-4-phosphate [E4P], and sedoheptulose7-phosphate [S7P]); organic acids (aconitate, citrate [Cit], fumarate [Fum], isocitrate [IsoCit], malate [Mal], oxaloacetate [OXA], 2-oxoglutarate [AKG], pyruvate, and succinate [Suc]); nucleotides (adenosine di- [ADP] and triphosphate [ATP]); coenzymes (acetyl-CoA [AcCoA], oxidized and reduced nicotinamide adenine dinucleotide $\left[\mathrm{NAD}^{+}\right.$and $\mathrm{NADH}$, respectively], oxidized and reduced nicotinamide adenine dinucleotide phosphate NADP $^{+}$ and NADPH, respectively]); and amino acids (alanine, [Ala], arginine [Arg], asparagine [Asn], aspartate [Asp], cysteine [Cys], glycine [Gly], glutamate [Glu], glutamine [Gln], histidine [His], isoleucine [Ile], leucine [Leu], lysine [Lys], methionine [Met], phenylalanine [Phe], proline [Pro], serine [Ser], threonine [Thr], tryptophan [Trp], tyrosine [Tyr], and valine [Val]). Metabolites were quantified as described previously [52] using an Agilent 1200 series MS and Agilent 6460 with Jet Stream Technology LC-MS/MS system (Agilent Technologies, Waldbronn, Germany) equipped with a Maestro C18 column $(2.1 \times 150 \mathrm{~mm}, 3-\mu \mathrm{m}$ particle size; Shimadzu, Kyoto, Japan).

\section{Extraction of metabolic intermediates}

For quantitative metabolomics, C. glutamicum cells were subjected to cold methanol quenching, as described previously [53], with slight modifications. A total of $15 \mathrm{~mL}$ of liquid culture at an $\mathrm{OD}_{600}$ of 2.0 was withdrawn from the medium and immediately sprayed into a $50 \mathrm{~mL}$ centrifugal tube (LMS Co., Ltd., Tokyo, Japan) containing double the volume of $40 \%(\mathrm{v} / \mathrm{v})$ aqueous methanol at $-25^{\circ} \mathrm{C}$. After sampling, the content of each tube was immediately mixed by vortexing for $5 \mathrm{~s}$ to quench cellular metabolism and subsequently centrifuged $\left(4000 \times g, 5 \mathrm{~min},-9^{\circ} \mathrm{C}\right)$. After decanting the supernatant, cell pellets were washed with $8 \mathrm{~mL}$ of $0.8 \%(\mathrm{w} / \mathrm{v}) \mathrm{NaCl}$ at $4{ }^{\circ} \mathrm{C}$ and centrifuged again $(4000 \times g$, $5 \mathrm{~min},-9^{\circ} \mathrm{C}$ ). After decanting the supernatant, the tubes containing the cell pellets were submerged directly into liquid nitrogen and stored at $-80^{\circ} \mathrm{C}$ until metabolite extraction. For metabolite extraction, $3.0 \mathrm{~mL}$ of cold methanol $\left(-25^{\circ} \mathrm{C}\right)$ containing (+)-camphor-10-sulfonic acid $\left(18 \mu \mathrm{g} \cdot \mathrm{L}^{-1}\right)$ was added to each tube as an internal standard for quantitative LC-MC/MS analysis. The tubes were vortexed for $30 \mathrm{~s}$, and the resulting cell suspensions were incubated at $-30{ }^{\circ} \mathrm{C}$ for $1 \mathrm{~h}$, after which $1.5 \mathrm{~mL}$ of the suspensions were transferred to $15 \mathrm{~mL}$ centrifuge tubes (LMS Co., Ltd.) containing $2.1 \mathrm{~mL}$ of chloroform and $1.5 \mathrm{~mL}$ of distilled water. These were then mixed by vortexing for $5 \mathrm{~s}$. After centrifuging the resulting suspensions at $15,000 \times g$ for $5 \mathrm{~min}$ at $4{ }^{\circ} \mathrm{C}$, the upper phases were transferred to new tubes and dried under vacuum. The dried samples were stored at $-80{ }^{\circ} \mathrm{C}$ until the metabolite analysis was performed.

\section{Statistical analysis}

Differences in sugar and metabolic intermediate concentrations and differences in cell density between the fermentation media were compared using the paired Student's $t$-test. Statistical significance was set at $p<0.05$.

\section{Abbreviations}

2PG + 3PG: 2- and 3-phosphoglycerate; 3,4-AHBA: 3-Amino-4-hydroxybenzoic acid; 6PG: 6-Phospho-D-glucono-1,5-lactone; AcCoA: Acetyl-CoA; ADP:

Adenosine 5'-diphosphate; AKG: 2-Oxoglutarate; Ala: Alanine; AMP: Adenosine 5'-monophosphate; Arg: Arginine; Asn: Asparagine; Asp: Aspartate; ATP: Adenosine 5'-triphosphate; Cit: Citrate; Cys: Cysteine; DCW: Dry cell weight; DHAP: Dihydroxyacetone phosphate; DO: Dissolved oxygen; E4P: Erythrose-4-phosphate; F6P: Fructose-6-phosphate; FBP: Fructose-1,6-bisphosphate; Fum: Fumarate; G6P: Glucose-6-phosphate; GAP: Glyceraldehyde-3-phosphate; GIn: Glutamine; Glu: Glutamate; Gly: Glycine; Ile: Isoleucine; IsoCit: Isocitrate; His: Histidine; LA: Lactate; L-DOPA: L-Dihydroxyphenylalanine; Leu: Leucine; Mal: Malate; Met: Methionine; $\mathrm{NAD}^{+}$: Oxidized nicotinamide adenine dinucleotide; $\mathrm{NADH}$ : Reduced nicotinamide adenine dinucleotide; NADP+ ${ }^{+}$Oxidized nicotinamide adenine dinucleotide phosphate; NADPH: Reduced nicotinamide adenine dinucleotide phosphate; OXA: Oxaloacetate; Phe: L-Phenylalanine; PEP: Phosphoenolpyruvate; PPP: Pentose phosphate pathway; Pro: Proline; Rib5P: Ribulose-5-phosphate; R5P: Ribose-5-phosphate; S7P: Sedoheptulose7-phosphate; Ser: Serine; Suc: Succinate; TCA: Tricarboxylic acid; Thr: Threonine; Trp: L-Tryptophan; Tyr: L-Tyrosine; Val: Valine; X5P: Xylulose-5-phosphate.

\section{Supplementary Information}

The online version contains supplementary material available at https://doi. org/10.1186/s12934-021-01714-z.

Additional file 1: Fig. S1. Cell growth of strain HKC5037 ( $\Delta / d h$ mutant) under oxygen limitation ( $\mathrm{DO}=0 \mathrm{ppm}$ ) in the absence or presence of urea in modified CGX II medium. The strain HKC5037 was grown under aerobic conditions until the late log phase in brain heart infusion medium, and the culture was then inoculated at an initial OD600 of 0.2 into modified CGX II medium containing glucose $(40 \mathrm{~g} / \mathrm{L})$ as the sole carbon source. During the cultivation, dissolved oxygen (DO) was controlled at 0 ppm using the DO-stat program.

\section{Acknowledgements}

We gratefully acknowledge Miho Iwami, Shoko Miyazaki, and Yoshimi Hori for their technical support.

\section{Authors' contributions}

$\mathrm{HK}$ and TH designed the research project, and carried out all the experiments and prepared the manuscript. ST, CO, and HK administered projects, and acquired funding. $Y O$ and $A K$ supervised the work and revised the manuscript. All authors read and approved the final manuscript.

\section{Funding}

This work was supported by JST CREST Grant Number JPMJCR13B3, JST-Mirai Program Grant Number JPMJMI17EG, and a KAKENHI Grant-in-Aid for Scientific Research (B) to H.K. (JP19KT0009) from the Japan Society for the Promotion of Science (JSPS). 


\section{Availability of data and materials}

All the data analyzed during this study have been included in this published article and Additional data.

\section{Declarations}

\section{Ethics approval and consent to participate}

Not applicable.

\section{Consent for publication}

Not applicable.

\section{Competing interests}

The authors declare no financial or commercial conflict of interest.

\section{Author details}

${ }^{1}$ Graduate School of Science, Technology and Innovation, Kobe University, 1-1 Rokkodai, Nada, Kobe 657-8501, Japan. ${ }^{2}$ Engineering Biology Research Center, Kobe University, 1-1 Rokkodai, Nada, Kobe 657-8501, Japan. ${ }^{3}$ Department of Biotechnology, Graduate School of Agricultural and Life Sciences, The University of Tokyo, 1-1-1, Yayoi, Bunkyo, Tokyo 113-8657, Japan. ${ }^{4}$ Collaborative Research Institute for Innovative Microbiology, The University of Tokyo, Bunkyo, Tokyo 113-8657, Japan. ${ }^{5}$ Bioscience and Biotechnology Center, Nagoya University, Furo, Chikusa, Nagoya 464-8601, Japan. ${ }^{6}$ Department of Chemical Science and Engineering, Graduate School of Engineering, Kobe University, 1-1 Rokkodai, Nada, Kobe 657-8501, Japan. ${ }^{7}$ Biomass Engineering Research Division, RIKEN, 1-7-22 Suehiro, Tsurumi, Yokohama, Kanagawa 230-0045, Japan.

Received: 22 September 2021 Accepted: 29 November 2021 Published online: 23 December 2021

\section{References}

1. Bongaerts J, Krämer M, Müller U, Raeven L, Wubbolts M. Metabolic engineering for microbial production of aromatic amino acids and derived compounds. Metab Eng. 2001;3:289-300. https://doi.org/10.1006/mben. 2001.0196.

2. Tateyama S, Masuo S, Suvannasara P, Oka Y, Miyazato A, Yasaki K, et al. Ultrastrong, transparent polytruxillamides derived from microbial photodimers. Macromol. 2016;49:3336-42. https://doi.org/10.1021/acs.macro mol.6b00220.

3. Kaneko T, Thi TH, Shi DJ, Akashi M. Environmentally degradable, highperformance thermoplastics from phenolic phytomonomers. Nat Mater. 2006:5:966-70. https://doi.org/10.1038/nmat1778.

4. Kawaguchi H, Katsuyama Y, Danyao D, Kahar P, Nakamura-Tsuruta S, Teramura $\mathrm{H}$, et al. Caffeic acid production by simultaneous saccharification and fermentation of kraft pulp using recombinant Escherichia coli. Appl Microbiol Biotechnol. 2017;101:5279-90. https://doi.org/10.1007/ s00253-017-8270-0.

5. Nakagawa A, Minami H, Kim JS, Koyanagi T, Katayama T, Sato F, et al. A bacterial platform for fermentative production of plant alkaloids. Nat Commun. 2011;2:326. https://doi.org/10.1038/Ncomms1327.

6. Noda S, Shirai T, Oyama S, Kondo A. Metabolic design of a platform Escherichia coli strain producing various chorismate derivatives. Metab Eng. 2016;33:119-29. https://doi.org/10.1016/j.ymben.2015.11.007.

7. McKenna R, Nielsen DR. Styrene biosynthesis from glucose by engineered E. coli. Metab Eng. 2011;13:544-54. https://doi.org/10.1016/j.ymben.2011. 06.005 .

8. Nag A, Ali MA, Kawaguchi H, Saito S, Kawasaki Y, Miyazaki S, et al. Ultrahigh thermoresistant lightweight bioplastics developed from fermentation products of cellulosic feedstock. Adv Sustain Syst. 2021;5:2000193. https://doi.org/10.1002/adsu.202000193.

9. Koma D, Yamanaka H, Moriyoshi K, Ohmoto T, Sakai K. Production of aromatic compounds by metabolically engineered Escherichia coli with an expanded shikimate pathway. Appl Environ Microbiol. 2012;78:6203-16. https://doi.org/10.1128/AEM.01148-12.

10. Suzuki H, Ohnishi Y, Furusho Y, Sakuda S, Horinouchi S. Novel benzene ring biosynthesis from $C_{3}$ and $C_{4}$ primary metabolites by two enzymes.
J Biol Chem. 2006;281:36944-51. https://doi.org/10.1074/jbc.M6081 03200.

11. Kawaguchi H, Sasaki K, Uematsu K, Tsuge Y, Teramura H, Okai N, et al. 3-Amino-4-hydroxybenzoic acid production from sweet sorghum juice by recombinant Corynebacterium glutamicum. Bioresour Technol. 2015;198:410-7. https://doi.org/10.1016/j.biortech.2015.09.024.

12. Limberg MH, Schulte J, Aryani T, Mahr R, Baumgart M, Bott M, et al. Metabolic profile of 1,5-diaminopentane producing Corynebacterium glutamicum under scale-down conditions: blueprint for robustness to bioreactor inhomogeneities. Biotechnol Bioeng. 2017;114:560-75. https://doi.org/10.1002/bit.26184.

13. Becker J, Klopprogge C, Zelder O, Heinzle E, Wittmann C. Amplified expression of fructose 1,6-bisphosphatase in Corynebacterium glutamicum increases in vivo flux through the pentose phosphate pathway and lysine production on different carbon sources. Appl Environ Microbiol. 2005;71:8587-96. https://doi.org/10.1128/Aem.71.12.8587-8596. 2005.

14. Chemler JA, Fowler ZL, McHugh KP, Koffas MAG. Improving NADPH availability for natural product biosynthesis in Escherichia coli by metabolic engineering. Metab Eng. 2010;12:96-104. https://doi.org/10.1016/j. ymben.2009.07.003.

15. Becker J, Zelder O, Häfner S, Schröder H, Wittmann C. From zero to hero: design-based systems metabolic engineering of Corynebacterium glutamicum for L-lysine production. Metab Eng. 2011;13:159-68. https://doi. org/10.1016/j.ymben.2011.01.003.

16. Xu JZ, Han M, Zhang JL, Guo YF, Zhang WG. Metabolic engineering Corynebacterium glutamicum for the L-lysine production by increasing the flux into L-lysine biosynthetic pathway. Amino Acids. 2014;46:2165-75. https://doi.org/10.1007/s00726-014-1768-1.

17. Kind S, Neubauer S, Becker J, Yamamoto M, Volkert M, von Abendroth G, et al. From zero to hero-production of bio-based nylon from renewable resources using engineered Corynebacterium glutamicum. Metab Eng. 2014;25:113-23. https://doi.org/10.1016/j.ymben.2014.05.007.

18. Kogure T, Kubota T, Suda M, Hiraga K, Inui M. Metabolic engineering of Corynebacterium glutamicum for shikimate overproduction by growtharrested cell reaction. Metab Eng. 2016;38:204-16. https://doi.org/10. 1016/j.ymben.2016.08.005.

19. Chen XZ, Li MM, Zhou L, Shen W, Algasan G, Fan Y, et al. Metabolic engineering of Escherichia coli for improving shikimate synthesis from glucose. Bioresour Technol. 2014;166:64-71. https://doi.org/10.1016/j. biortech.2014.05.035.

20. Kiefer P, Heinzle E, Zelder O, Wittmann C. Comparative metabolic flux analysis of lysine-producing Corynebacterium glutamicum cultured on glucose or fructose. Appl Environ Microbiol. 2004;70:229-39. https://doi. org/10.1128/Aem.70.1.229-239.2004.

21. Sun Y, Guo WW, Wang F, Yang YK, Dai XF, Liu XX, et al. Transcriptome and multivariable data analysis of Corynebacterium glutamicum under different dissolved oxygen conditions in bioreactors. PLOS ONE. 2016;11:17. https://doi.org/10.1371/journal.pone.0167156.

22. Liu XX, Yang S, Wang F, Dai XF, Yang YK, Bai ZH. Comparative analysis of the Corynebacterium glutamicum transcriptome in response to changes in dissolved oxygen levels. J Ind Microbiol Biotechnol. 2017;44:181-95. https://doi.org/10.1007/s10295-016-1854-3.

23. Inui M, Suda M, Okino S, Nonaka H, Puskas LG, Vertes AA, et al. Transcriptional profiling of Corynebacterium glutamicum metabolism during organic acid production under oxygen deprivation conditions. Microbiol. 2007;153:2491-504. https://doi.org/10.1099/mic.0.2006/005587-0.

24. Koffas MAG, Jung GY, Aon JC, Stephanopoulos G. Effect of pyruvate carboxylase overexpression on the physiology of Corynebacterium glutamicum. Appl Environ Microbiol. 2002;68:5422-8. https://doi.org/10. 1128/AEM.68.11.5422-5428.2002.

25. Liu SP, Xiao MR, Zhang L, Xu J, Ding ZY, Gu ZH, et al. Production of L-phenylalanine from glucose by metabolic engineering of wild type Escherichia coli W3110. Process Biochem. 2013;48:413-9. https://doi.org/ 10.1016/j.procbio.2013.02.016.

26. Yoshida A, Tomita T, Kurihara T, Fushinobu S, Kuzuyama T, Nishiyama M. Structural insight into concerted inhibition of $a_{2} \beta_{2}$-type aspartate kinase from Corynebacterium glutamicum. J Mol Biol. 2007;368:521-36. https:// doi.org/10.1016/j.jmb.2007.02.017. 
27. Ensari S, Kim JH, Lim HC. Unstructured model for L-lysine fermentation under controlled dissolved oxygen. Biotechnol Progr. 2003;19:1387-90. https://doi.org/10.1021/bp034019s.

28. Peng ZJ, Fang J, Li JH, Liu L, Du GC, Chen J, et al. Combined dissolved oxygen and $\mathrm{pH}$ control strategy to improve the fermentative production of L-isoleucine by Brevibacterium lactofermentum. Bioproc Biosyst Eng. 2010;33:339-45. https://doi.org/10.1007/s00449-009-0329-6.

29. Shu CH, Liao CC. Optimization of L-phenylalanine production of Corynebacterium glutamicum under product feedback inhibition by elevated oxygen transfer rate. Biotechnol Bioeng. 2002;77:131-41. https://doi.org/10.1002/bit.10125

30. Zhao CG, Cheng LK, Xu QY, Wang J, Shen ZQ, Chen N. Improvement of the production of L-tryptophan in Escherichia coli by application of a dissolved oxygen stage control strategy. Ann Microbiol. 2016;66:843-54. https://doi.org/10.1007/s13213-015-1172-4.

31. Kim B, Binkley R, Kim HU, Lee SY. Metabolic engineering of Escherichia coli for the enhanced production of L-tyrosine. Biotechnol Bioeng 2018;115:2554-64. https://doi.org/10.1002/bit.26797.

32. Shen J, Chen J, Solem C, Jensen PR, Liu JM. Disruption of the oxidative pentose phosphate pathway stimulates high-yield production using resting Corynebacterium glutamicum in the absence of external electron acceptors. Appl Environ Microbiol. 2020;86:e02114-02120. https://doi. org/10.1128/AEM.02114-20.

33. Hua Q, Shimizu K. Effect of dissolved oxygen concentration on the intracellular flux distribution for pyruvate fermentation. J Biotechnol. 1999;68:135-47. https://doi.org/10.1016/s0168-1656(98)00196-5.

34. Shinfuku Y, Sorpitiporn N, Sono M, Furusawa C, Hirasawa T, Shimizu H. Development and experimental verification of a genome-scale metabolic model for Corynebacterium glutamicum. Microb Cell Fact. 2009. https://doi.org/10.1186/1475-2859-8-43.

35. Bott $M$, Niebisch $A$. The respiratory chain of Corynebacterium glutamicum. J Biotechnol. 2003;104:129-53. https://doi.org/10.1016/S0168-1656(03) 00144-5.

36. Molenaar D, van der Rest ME, Drysch A, Yücel R. Functions of the membrane-associated and cytoplasmic malate dehydrogenases in the citric acid cycle of Corynebacterium glutamicum. J Bacteriol. 2000;182:6884-91. https://doi.org/10.1128/jb.182.24.6884-6891.2000.

37. Bartek T, Blombach B, Zönnchen E, Makus P, Lang S, Eikmanns BJ, et al. Importance of NADPH supply for improved L-valine formation in Corynebacterium glutamicum. Biotechnol Progr. 2010;26:361-71. https:// doi.org/10.1002/btpr.345.

38. Chen XW, Alonso AP, Allen DK, Reed JL, Shachar-Hill Y. Synergy between 13C-metabolic flux analysis and flux balance analysis for understanding metabolic adaption to anaerobiosis in E. coli. Metab Eng. 2011;13:38-48. https://doi.org/10.1016/j.ymben.2010.11.004.

39. Marienhagen J, Eggeling L. Metabolic function of Corynebacterium glutamicum aminotransferases AlaT and AvtA and impact on L-valine production. Appl Environ Microbiol. 2008;74:7457-62. https://doi.org/10. 1128/AEM.01025-08.

40. Vogt M, Krumbach K, Bang WG, van Ooyen J, Noack S, Klein B, et al. The contest for precursors: channelling L-isoleucine synthesis in Corynebacterium glutamicum without byproduct formation. Appl Microbiol Biotechnol. 2015;99:791-800. https://doi.org/10.1007/s00253-014-6109-5

41. Inui M, Murakami S, Okino S, Kawaguchi H, Vertès AA, Yukawa H. Metabolic analysis of Corynebacterium glutamicum during lactate and succinate productions under oxygen deprivation conditions. J Mol Microb Biotechnol. 2004;7:182-96. https://doi.org/10.1159/000079827.

42. Yasuda K, Jojima T, Suda M, Okino S, Inui M, Yukawa H. Analyses of the acetate-producing pathways in Corynebacterium glutamicum under oxygen-deprived conditions. Appl Microbiol Biotechnol. 2007;77:853-60. https://doi.org/10.1007/s00253-007-1199-y.

43. Sambrook J, Russell DW. Molecular cloning: a laboratory manual. 3rd ed. New York: Cold Spring Harbor Laboratory Press; 2001.

44. Keilhauer C, Eggeling L, Sahm H. Isoleucine synthesis in Corynebacterium glutamicum: molecular analysis of the ilvB-ilvN-ilvC operon. J Bacteriol. 1993;175:5595-603. https://doi.org/10.1128/jb.175.17.5595-5603.1993.

45. Vertès AA, Inui M, Kobayashi M, Kurusu Y, Yukawa H. Presence of mrr-like and $\mathrm{mcr}$-like restriction systems in coryneform bacteria. Res Microbiol. 1993;144:181-5. https://doi.org/10.1016/0923-2508(93)90043-2.

46. Kawaguchi H, Sasaki M, Vertes AA, Inui M, Yukawa H. Engineering of an L-arabinose metabolic pathway in Corynebacterium glutamicum.
Appl Microbiol Biotechnol. 2008;77:1053-62. https://doi.org/10.1007/ s00253-007-1244-x

47. Kawaguchi H, Sazuka T, Kondo A. Complete and draft genome sequences of amino acid-producing Corynebacterium glutamicum strains ATCC 21799 and ATCC 31831 and their genomic islands. Microbiol Resour. 2020;9: e00430-00420. https://doi.org/10.1128/MRA.00430-20.

48. Inui M, Kawaguchi $H$, Murakami S, Vertes AA, Yukawa H. Metabolic engineering of Corynebacterium glutamicum for fuel ethanol production under oxygen-deprivation conditions. J Mol Microbiol Biotechnol. 2004;8:243-54. https://doi.org/10.1159/000086705.

49. Kawaguchi H, Miyagawa H, Nakamura-Tsuruta S, Takaya N, Ogino C, Kondo A. Enhanced phenyllactic acid production in Escherichia coli via oxygen limitation and shikimate pathway gene expression. Biotechnol J. 2019;14:1800478. https://doi.org/10.1002/biot.201800478.

50. Kawaguchi H, Yoshihara K, Hara KY, Hasunuma T, Ogino C, Kondo A. Metabolome analysis-based design and engineering of a metabolic pathway in Corynebacterium glutamicum to match rates of simultaneous utilization of D-glucose and L-arabinose. Microb Cell Fact. 2018;17:76. https://doi.org/10.1186/s12934-018-0927-6.

51. Buescher JM, Moco S, Sauer U, Zamboni N. Ultrahigh performance liquid chromatography-tandem mass spectrometry method for fast and robust quantification of anionic and aromatic metabolites. Anal Chem. 2010;82:4403-12. https://doi.org/10.1021/Ac100101d.

52. Kato H, Izumi Y, Hasunuma T, Matsuda F, Kondo A. Widely targeted metabolic profiling analysis of yeast central metabolites. J Biosci Bioeng 2012;113:665-73. https://doi.org/10.1016/j.jbiosc.2011.12.013.

53. de Jonge LP, Douma RD, Heijnen JJ, van Gulik WM. Optimization of cold methanol quenching for quantitative metabolomics of Penicillium chrysogenum. Metabol. 2012;8:727-35. https://doi.org/10.1007/ s11306-011-0367-3.

\section{Publisher's Note}

Springer Nature remains neutral with regard to jurisdictional claims in published maps and institutional affiliations.

Ready to submit your research? Choose BMC and benefit from

- fast, convenient online submission

- thorough peer review by experienced researchers in your field

- rapid publication on acceptance

- support for research data, including large and complex data types

- gold Open Access which fosters wider collaboration and increased citations

- maximum visibility for your research: over $100 \mathrm{M}$ website views per year

At BMC, research is always in progress.

Learn more biomedcentral.com/submissions 